\title{
PPAR $\gamma$ agonists promote the resolution of myelofibrosis in preclinical models
}

\author{
Juliette Lambert, ${ }^{1,2,3}$ Joseph Saliba, ${ }^{1}$ Carolina Calderon, ${ }^{1,3}$ Karine Sii-Felice, ${ }^{1}$ Mohammad Salma, ${ }^{4,5}$ Valérie Edmond, ${ }^{6}$ \\ Jean-Claude Alvarez, ${ }^{7,8}$ Marc Delord, ${ }^{9}$ Caroline Marty, ${ }^{6}$ Isabelle Plo, ${ }^{6}$ Jean-Jacques Kiladjian, ${ }^{3,10}$ Eric Soler, ${ }^{4,5}$ \\ William Vainchenker, ${ }^{6}$ Jean-Luc Villeval, ${ }^{6}$ Philippe Rousselot, ${ }^{1,2,3,11}$ and Stéphane Prost ${ }^{1,3}$
}

'Division of Innovative Therapies, CEA/DRF/François Jacob Biology Institute, UMR1184 IMVA-HB/IDMIT, Université Paris-Saclay, Fontenay-aux-Roses, France. Department of Hematology and Oncology,

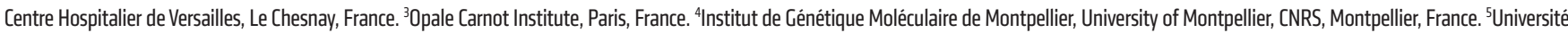
de Paris, Laboratory of Excellence GR-Ex, Paris, France. ${ }^{6}$ INSERM, UMR1287, Université Paris-Saclay, Gustave Roussy, Villejuif, France. `Département de Pharmacologie-Toxicologie, Hôpitaux Universitaires Paris Ile-de-France Ouest, AP-HP, Hôpital Raymond-Poincaré, FHU Sepsis, Garches, France. ${ }^{8}$ MasSpecLab, Plateforme de spectrométrie de masse, INSERM U-1173, Université Paris-Saclay (Versailles Saint-Quentin-en-Yvelines), UFR des sciences de la santé, Montigny-le-Bretonneux, France. ${ }^{9}$ Recherche Clinique, Centre Hospitalier de Versailles, Le Chesnay, France. ${ }^{10}$ Université de Paris, AP-HP, Hôpital Saint-Louis, Centre d'Investigations Cliniques CIC 1427, INSERM, Paris, France. "Université Paris-Saclay (Versailles Saint-Quentin-en-Yvelines), UFR des sciences de la santé, Montigny-le-Bretonneux, France.

\begin{abstract}
Myelofibrosis (MF) is a non-BCR-ABL myeloproliferative neoplasm associated with poor outcomes. Current treatment has little effect on the natural history of the disease. MF results from complex interactions between (a) the malignant clone, (b) an inflammatory context, and (c) remodeling of the bone marrow (BM) microenvironment. Each of these points is a potential target of PPAR $\gamma$ activation. Here, we demonstrated the therapeutic potential of PPAR $\gamma$ agonists in resolving MF in 3 mouse models. We showed that PPAR $\gamma$ agonists reduce myeloproliferation, modulate inflammation, and protect the BM stroma in vitro and ex vivo. Activation of PPAR $\gamma$ constitutes a relevant therapeutic target in MF, and our data support the possibility of using PPAR $\gamma$ agonists in clinical practice.
\end{abstract}

\section{Introduction}

BCR-ABL-negative myeloproliferative neoplasms (MPNs) arise from mutated hematopoietic stem cells (HSCs) that clonally expand and include polycythemia vera (PV), essential thrombocythemia (ET), and primary myelofibrosis (1). They are driven by the acquisition of somatic mutations, mainly in the JAK2, MPL, and $C A L R$ genes $(2,3)$ in a founding HSC, all leading to constitutive activation of the JAK/STAT signaling pathway. A common feature of MPNs is an initial hypercellular phase with clonal expansion of the myeloid lineages, followed in a later phase by megakaryocyte hyperplasia and bone marrow (BM) fibrosis, two main characteristics of myelofibrosis (MF). Presenting either as primary or as secondary evolving from PV or ET, MF leads to BM and blood cell production failure revealed by severe anemias and cytopenias and associated with an extramedullary hematopoiesis, inflammation, and splenomegaly. Patients with severe MF have a poor prognosis with disease-related morbidity due to complications, such as thrombosis, hemorrhaging, infections, and transformation to acute leukemia (4). This results in a poor quality of life with a short median survival of approximately 6 years (5). Histopathological examination shows MF to be characterized by the gradually increasing deposition of reticulin and collagen fibers, thickening and distortion of bone trabeculae, and megakaryocytic

Conflict of interest: The authors have declared that no conflict of interest exists. Copyright: () 2021, American Society for Clinical Investigation.

Submitted: January 23, 2020; Accepted: April 22, 2021; Published: June 1, 2021.

Reference information: J Clin Invest. 2021;131(11):e136713.

https://doi.org/10.1172/JCl136713. hyperplasia with atypical features (6). The role of increased JAK/ STAT signaling in the proliferation of the neoplastic clone and the role of deregulated proinflammatory cytokine expression in driving fibrosis appear to be 2 major pathogenic processes and putative therapeutic targets that contribute to the initiation and progression of MF. Although allogenic stem cell transplantation leads to remission, it is associated with substantial treatment-related morbidity and mortality and is essentially restricted to younger patients (7). Currently, treatment options are mainly palliative and directed toward improvement of disease symptoms such as splenomegaly, constitutional symptoms, and anemia (8). The emergence of JAK2 inhibitors has provided clinical benefit for splenomegaly and constitutional symptoms. However, MF patients enrolled in the pivotal phase III COMFORT study testing ruxolitinib failed to show improvement in histopathological abnormalities after 6 and 12 months of therapy or in incidence of leukemic transformation (9-11). Long-term follow-up showed some survival advantage for patients treated with ruxolitinib, but anemia and thrombocytopenia might be worsened by the treatment and are still a challenge in the management of MF (12), and around $50 \%$ of patients stopped treatment because of cytopenia $(12,13)$. PEGylated IFN- $\alpha$ appears to be a potential alternative therapy, with a reduction of allele burden by more than $50 \%$ in $58.8 \%$ of patients and a significant increase in survival, which correlates with the duration of PEGylated IFN therapy (14). The complex pathogenesis of MF cannot be modeled in vitro, but MPN mouse models are an invaluable tool for further characterization of the disease mechanisms, as well as for testing novel therapeutic agents (15-18). Mice of the TPO ${ }^{\text {high }}$ model show a plasma thrombopoietin (TPO) level approximately 
10,000-fold higher than that in WT mice or in lethally irradiated recipients with transplanted BM from WT mice (data not shown). Mice develop a myeloproliferative disorder with megakaryocytic proliferation, extramedullary hematopoiesis, osteosclerosis, and severe MF approximately 3 months after transplantation (17). Mice of the JAK2 ${ }^{\mathrm{V} 617 \mathrm{~F}}$ model develop a disease that mimics human PV, evolving into severe MF 5 months after transplantation (15). In the CALRdel52 retroviral model, mice develop an ET-like phenotype, evolving to MF after 5 months (16).

Unmet needs include novel therapeutics or combination of antifibrogenic strategies with currently available treatments to modify the effects of the natural history of MF and to control the 3 components of the disease, the neoplastic clone, the inflammatory context, and the remodeling of the BM microenvironment. Each of these points is a potential target of PPAR $\gamma$ activation.

Peroxisome proliferator-activated receptor- $\gamma$ (PPAR $\gamma$; NR1C3) is a member of the nuclear hormone receptor superfamily of ligand-activated transcription factors. Its pivotal role has been demonstrated in glucose homeostasis, lipid metabolism, cell growth, and, posteriorly, inflammation and the regulation of connective tissue biology (19). We previously reported that PPAR $\gamma$ is a negative regulator of the STAT5 pathway, impairing stressinduced hematopoiesis (20) and eroding the chronic myeloid leukemia stem cell pool in biological and clinical assays $(21,22)$. In addition, PPAR $\gamma$ agonists play an important role in dampening inflammation (23) and attenuate synthesis of proinflammatory cytokines by macrophages/monocytes $(24,25)$ and megakaryocytes (26). An inverse relationship between fibrosis and PPAR $\gamma$ expression/function has been reported in multiple human fibrosing disorders and animal models of fibrosis (27). However, mechanistic insights are still needed, and to our knowledge no study has tested the antifibrotic potential of PPAR $\gamma$ in MF. As PPAR $\gamma$ may act on both myeloproliferation and inflammation, as well as fibrosis, we focused on these 3 master components of the pathophysiology of the disease, with an emphasis on the therapeutic potential of PPAR $\gamma$ agonists in MF.

Transforming growth factor- $\beta$ (TGF- $\beta$ ) is the main regulator of physiological fibrogenesis and pathological fibrosis (28-30), and has emerged as a major therapeutic target in fibrotic diseases (29), especially MF (31). TGF- $\beta$ is found in 3 isoforms: TGF- $\beta 1$, TGF- $\beta 2$, and TGF- $\beta 3$. TGF- $\beta 1$ is the most abundant of the three, and platelets, megakaryocytes, and $\mathrm{BM}$ are the major sources of TGF- $\beta 1$ (32). The involvement of TGF- $\beta$ levels was first described in patients with acute megakaryoblastic leukemia, which is a particular form of leukemia associated with BM fibrosis (33), and similar observations were then reported in MF patients (34). Mouse models of MF, including the TPO ${ }^{\text {high }}$ and GATA $1^{\text {low }}$ models, have provided important insights into the major role of TGF- $\beta$ in the pathogenesis of fibrosis (35).

Here, we show that treatment with PPAR $\gamma$ agonists (including pioglitazone) counteracts MF-related anemia, represses leukocytosis, and improves $\mathrm{BM}$ and splenic changes related to the disease in 3 preclinical mouse models of MF. We demonstrate that PPAR $\gamma$ activation represses the survival and clonogenicity of cells from cell lines and PV/MF patients and slows cell proliferation in vitro and in PV (JAK2 ${ }^{\mathrm{V} 617 \mathrm{~F}}$ ) and ET (CALRdel52) mouse models. We also assess the potential effect of PPAR $\gamma$ agonists on TGF- $\beta 1$-driven fibrosis via their antagonism of regulation of the TGF- $\beta$ signaling pathway.
Using genome-wide assays, we show that the effect of PPAR $\gamma$ agonists is mainly mediated via a competition for the transcriptional cofactor p300. Overall, these results establish that PPAR $\gamma$ agonists may be novel therapeutic candidates for MPNs with MF.

\section{Results}

PPAR $\gamma$ activation counteracts $M F$ and prevents anemia, cytopenia, and BM hypocellularity in mouse MF models. We investigated the ability of PPAR $\gamma$ agonists to counteract MF and MF-related symptoms by treating 3 different mouse models of MF (TPO ${ }^{\text {high }}$, $\mathrm{JAK}^{\mathrm{V} 617 \mathrm{~F}}$, and CALRdel52, respectively presented in Figure 1, $\mathrm{E}-\mathrm{G}$ ) with pioglitazone or mesalazine. Drugs were given orally in pellets, and after 3 weeks of treatment, the plasmatic levels were $5336 \pm 1364 \mathrm{ng} / \mathrm{mL}(13.6 \pm 3.4 \mu \mathrm{M})$ and $344 \pm 199 \mathrm{ng} / \mathrm{mL}(2.3 \pm 1.3$ $\mu \mathrm{M})$ for pioglitazone and mesalazine, respectively.

First, JAK $2^{\mathrm{V} 617 \mathrm{~F}}$ mice were treated with the PPAR $\gamma$ agonist pioglitazone or mesalazine. Histology of the organs by H\&E staining at week 37 (W37) after transplantation showed that treatment with PPAR $\gamma$ agonists preserved BM and spleen cellularity (Figure 1, A and $\mathrm{C}$ ) and reduced myeloid invasion of the spleen, which retained a nearly normal white pulp architecture (Figure 1C and Table 1). Silver staining showed a reduction of reticular fibers in femoral BM and spleen (Figure 1, B and D), which was illustrated by a decrease of fibrosis graduation in both BM and spleen (Table 1). Osteosclerosis was almost abolished by the PPAR $\gamma$ agonists, and the treated mice also had significantly smaller spleens (Table 1 ).

Second, we monitored the hemoglobin level in the 3 mouse models of MF, TPO ${ }^{\text {high }}$ (Figure 1E), post-PV (JAK2 ${ }^{\mathrm{V} 617 \mathrm{~F}}$; Figure $1 \mathrm{~F}$ ), and post-ET (CALRdel52; Figure 1G), treated or not with pioglitazone or mesalazine. The PPAR $\gamma$ agonists abrogated the MF-driven decrease of hemoglobin in all 3 models, despite differences in the kinetics of MF (Figure 1, E-G, and Supplemental Figure 1, A-F, including red blood cell counts; supplemental material available online with this article; https://doi.org/10.1172/JCI136713DS1). We observed this effect even when the treatment was initiated during disease progression, as in the MF-JAK2 ${ }^{\mathrm{V} 617 \mathrm{~F}}$ model treated at W20 after transplantation (Figure $1 \mathrm{~F}$ and Supplemental Figure 1, B and E). We also observed a reduction of thrombocytosis in the treated mice of the $3 \mathrm{MF}$ models (Supplemental Figure 1, G-I), and the number of megakaryocytes quantified after von Willebrand factor staining was strikingly reduced in both the BM and the spleen in the JAK2 ${ }^{\mathrm{V} 617 \mathrm{~F}}$ model (Supplemental Figure 2, A and $\mathrm{B})$. We recently reported megakaryocyte (MK) hyperplasia in CALRdel52 mouse models (36). We confirmed here that the size of MKs is particularly high in this model, and we observed that pioglitazone treatment restored a normal MK size and also led to a significant reduction (2.2-fold) in the density of BM MKs, suggesting an effect on MK proliferation and hyperplasia (Supplemental Figure 2C). In the JAK2 ${ }^{\mathrm{V} 617 \mathrm{~F}}$ model, where the size of the MKs is less increased, PPAR $\gamma$ activation induced, to a lesser degree, a reduction in the size of the MKs in the BM and spleen (Supplemental Figure 2, A and B). These results were associated with a significant conservation of femoral BM cellularity, with a more than 2-fold increase in the total number of CD 45.2 hematopoietic cells after treatment as compared with untreated control mice (Figure 1, H, $\mathrm{J}$, and L). Notably, although hemoglobin levels were preserved in the presence of pioglitazone in the CALRdel52 MF model, BM cel- 
A
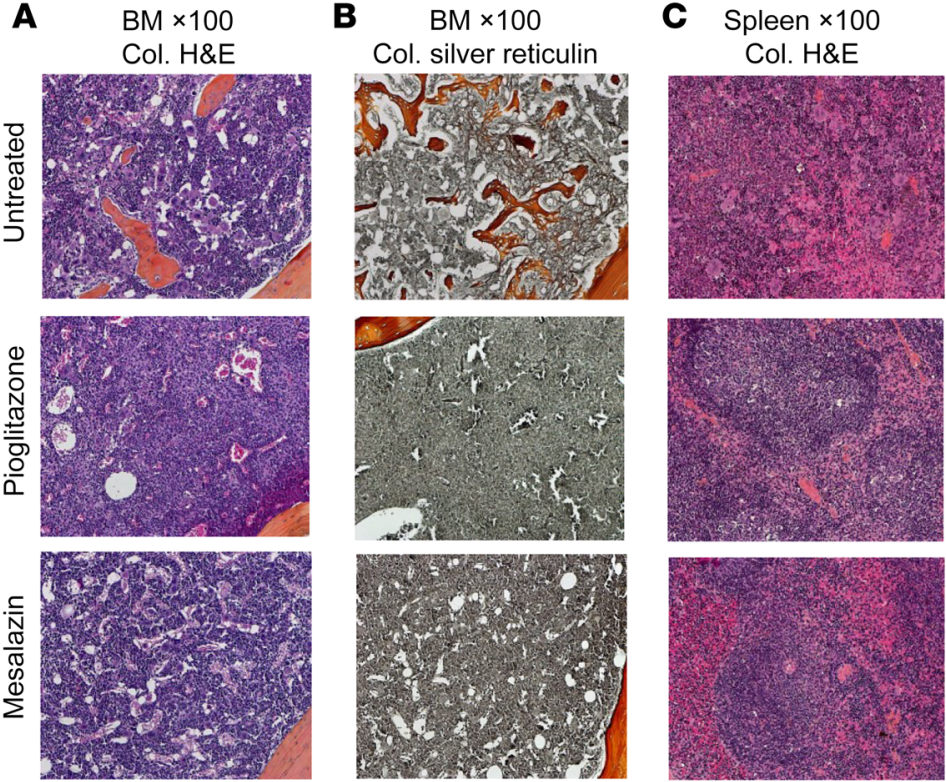

$\mathbf{F}$
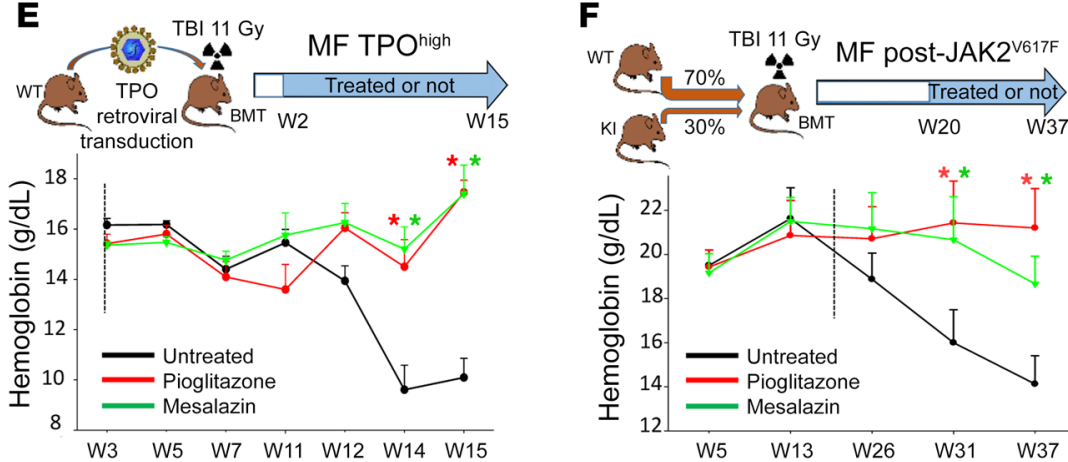

G
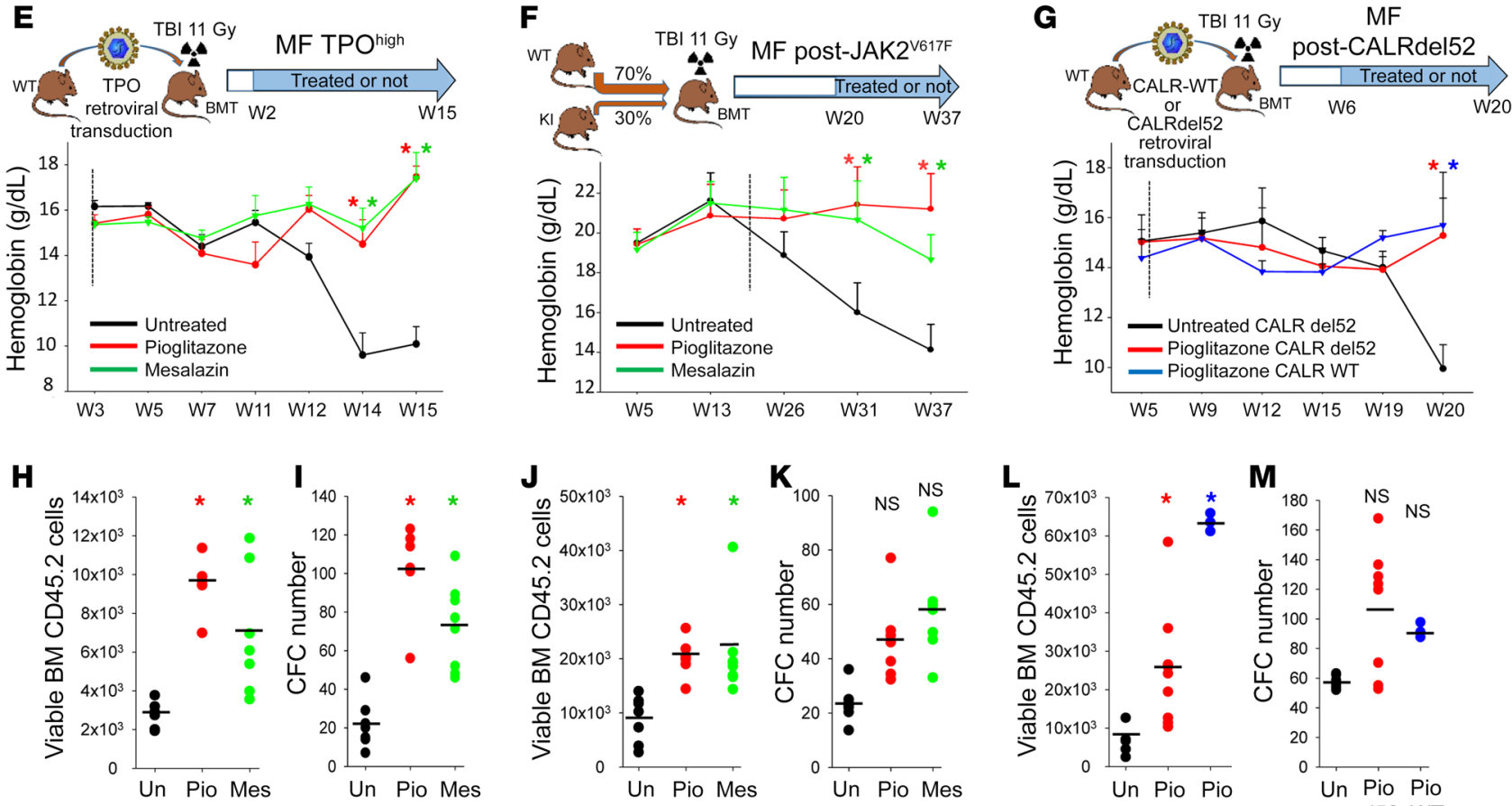

D Spleen $\times 100$
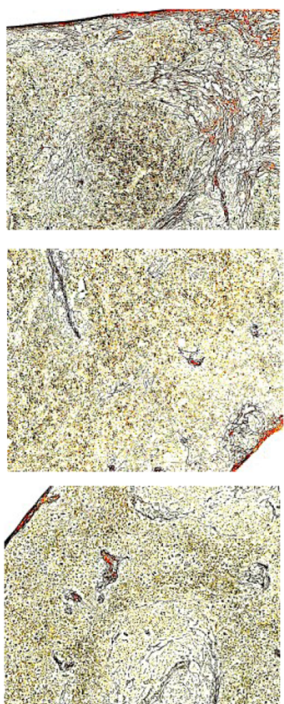

Col. silver reticulum

Figure 1. Treatment with PPAR $\gamma$ agonists prevents MF in 3 murine models of MPN. Images present the histological examination of the BM (femur) and spleen of 1 representative mouse per treatment group in the MF post-JAK2 ${ }^{\mathrm{V} 617 \mathrm{~F}}$ model (W37). (A and $\left.\mathbf{C}\right) \mathrm{H} \& \mathrm{E}$ coloration shows BM and spleen cellularity, respectively. (B and $\mathbf{D})$ Silver reticulin coloration shows a dense network of reticulin fibers in the BM and spleens of untreated animals, whereas only scattered fibers are seen in the BM and spleens of mice treated with pioglitazone or mesalazine. (Original magnification, $\times 100$.) (E-C) PPAR $\gamma$ agonists counteract the decrease of hemoglobin levels related to the development of BM fibrosis in the MF TPO ${ }^{\text {high }}$ model (E), MF post-JAK2 ${ }^{\mathrm{V} 617 F}$ model (F), and MF post-CALRdel52 model (C). The black vertical dashed lines indicate initiation of treatment. (H-M) PPAR $\gamma$ agonists prevent BM hematopoietic cell depletion in the MF TPO ${ }^{\text {high }}$ model (H), MF post-JAK2 ${ }^{\mathrm{V} 617 \mathrm{~F}}$ model (J), and MF post-CALRdel52 model (L) and preserve the clonogenic potential of BM hematopoietic progenitors in the MF TPO ${ }^{\text {high }}$ model (I), MF post-JAK2 ${ }^{\mathrm{V} 617}$ model (K), and MF post-CALRdel52 model (M). The black horizontal lines represent the mean. ${ }^{*}$ Statistically significant difference $(P<0.05)$. BMT, bone marrow transplantation; CFC, colony-forming cell; d52, CALRdel52; Mes, mesalazine; Pio, pioglitazone; TBI, total-body irradiation; Un, untreated; W, week; WT, CALR-WT. ( $n=10$ mice per condition, except CALR-WT pioglitazone $n=5$ ).

lularity was significantly lower than that of CALR-WT littermates, suggesting that the effect of PPAR $\gamma$ on MF inhibition is not complete (Figure 1L). The number of colony-forming cells from treated mice was 2-fold higher in the JAK2 ${ }^{\mathrm{V} 617 \mathrm{~F}} \mathrm{MF}$ (Figure $1 \mathrm{~K}$ ) and MF
post-CALRdel52 (Figure 1M) models and more than 4-fold higher in the $\mathrm{TPO}^{\text {high }}$ model than that from untreated mice $(P<0.05$; Figure 1I), suggesting that the clonogenic potential in myeloid progenitors was better in treated mice. 
Table 1. Histopathological analysis of the BM (femur) and spleen in the MF post-JAK2 ${ }^{\mathrm{V} 617 \mathrm{~F}}$ murine model (W37 after transplantation)

\begin{tabular}{|c|c|c|c|c|c|c|c|}
\hline & \multirow[t]{2}{*}{ Number } & \multicolumn{2}{|c|}{ BM analysis } & \multicolumn{4}{|c|}{ Spleen analysis } \\
\hline & & Fibrosis & Osteosclerosis & Fibrosis & Structure & Mass (mg) & Mean \pm SD \\
\hline \multirow{3}{*}{ Untreated } & 962 & Grade $3 / 3$ & +++ & Grade $2-3 / 3$ & $M \gg>L$ & 552 & \\
\hline & 896 & Grade 1-2/3 & Absence & Grade 0/3 & $\approx$ Normal & 475 & \\
\hline & 897 & Grade 1-2/3 & Absence & Grade $1 / 3$ & $M \gg>L$ & 486 & \\
\hline \multirow[t]{4}{*}{ Pioglitazone } & 964 & Grade 2-3/3 & $+(+)$ & Grade 1.75/3 & $M>L$ & 538 & \\
\hline & 966 & Grade $0 / 3$ & Absence & Grade $0-1 / 3$ & $\approx$ Normal & 109 & $320 \pm 204$ \\
\hline & 898 & Grade 0-1/3 & Absence & Grade 1-2/3 & $\approx$ Normal & 435 & \\
\hline & 899 & Grade 0/3 & Absence & Grade $0-1 / 3$ & $\approx$ Normal & 94 & \\
\hline \multirow[t]{3}{*}{ Mesalazine } & 967 & Grade $0 / 3$ & Absence & Grade $1.25 / 3$ & $\approx$ Normal & 246 & \\
\hline & 968 & Grade $0-1 / 3$ & Absence & Grade $1.75 / 3$ & $\approx$ Normal & 175 & \\
\hline & 969 & Grade $0 / 3$ & Absence & Grade 1.5/3 & $\approx$ Normal & 83 & $304 \pm 196$ \\
\hline
\end{tabular}

The table recapitulates the histopathological examination of BM and spleen of 5 representative mice per treatment group (W37 after transplantation). BM fibrosis and spleen fibrosis were graded from 0 to 3,3 being the highest grade of fibrosis. Osteosclerosis was estimated using a semiquantitative scale from + to +++. Absence denotes no osteosclerosis. Spleen architecture was assessed by myeloid cell invasion (M) to the detriment of lymphoid cells (L). The mass of the spleens is expressed in milligrams, and the mean \pm SD per group is presented. ${ }^{A} P=0.0317$ vs. Piogliazone. ${ }^{B} P=0.0317$ vs. Mesalazine.

Overall, these results strongly support the therapeutic effect of PPAR $\gamma$ ligands in the control of MF. We next assessed the impact of PPAR $\gamma$ activation on proliferation, inflammation, and the induction of $\mathrm{BM}$ stromal fibrosis to decipher the mechanisms by which PPAR $\gamma$ may affect MF development.

PPAR agonists slow the myeloproliferative effect driven by the neoplastic clone. It has become increasingly clear over the last couple of years that at least 2 distinct pathogenic processes contribute to the initiation and progression of MF: (a) stem cell-derived clonal myeloproliferation and (b) reactive cytokine-driven fibrosis. We assessed whether PPAR $\gamma$ activation may affect myeloproliferation driven by the malignant hematopoietic clone in vivo, by analyzing the hematological consequences of pioglitazone treatment in PV-JAK2 ${ }^{\mathrm{V} 617 \mathrm{~F}}$-GFP and ET-CALRdel52-GFP mouse models. We focused on early stages of the disease in each model to avoid a potential impact of BM fibrosis. Pioglitazone treatment initiated at W6 after transplantation significantly reduced the JAK2 ${ }^{\mathrm{V} 617 \mathrm{~F}}$-driven polycythemia, with a reduction of the hematocrit values: $82 \%$ vs. $73 \%$ at $\mathrm{W} 10,86 \%$ vs. $76 \%$ at W14, and $86 \%$ vs. $75 \%$ at W23 in treated versus untreated mice, respectively ( $P \leq 0.05$; Figure $2 A)$. Pioglitazone also limited CALRdel52-driven thrombocytosis, with a reduction in platelet number of $49 \%$ at W9 and $40 \%$ at W12 $(P \leq 0.05)$ and $38 \%$ at W15 (NS; Figure 2B). However, pioglitazone failed to completely normalize the hematocrit in the PV model or the platelet count in the ET model (Figure 2, A and B). Similarly, the early administration of PPAR $\gamma$ agonists significantly reduced the platelet count in the TPO $^{\text {high }}$ model (W7 to W12; Supplemental Figure 1G).

We assessed the effect of pioglitazone on expansion of the mutated clone, by quantifying the proportion of GFP cells. There was a slight reduction in the number of CALRdel52-GFP cells in the CD45.2 hematopoietic compartment (Figure 2D). This trend was more evident for myeloid cells (CD11b cells; Figure 2F). Pioglitazone was able to significantly reduce the proportion of JAK2 $2^{\mathrm{V} 617 \mathrm{~F}_{-}}$ GFP cells in the PV mouse model, in both the CD45.2 peripheral blood compartment (Figure 2C) and the CD11b population, with greater effect, due to the enrichment of JAK2 ${ }^{\mathrm{V} 617 \mathrm{~F}}$ in the myeloid compartment (Figure 2E). In the BM, treated animals showed an increase in the number of stem cells ( $\left.\mathrm{Lin}^{-} \mathrm{Sca}^{+} \mathrm{Kit}^{+}[\mathrm{LSK}]\right)$, notably in the short-term stem cell compartment (ST-HSC, LSK-CD34+; Supplemental Figure $3 \mathrm{~A}$ ), and this effect was associated with a decrease in the proportion of JAK $2^{\mathrm{V} 617 \mathrm{~F}}$-GFP cells in the LSK compartment, more particularly in the ST-HSC (Supplemental Figure 3B).

We assessed the effect of the PPAR $\gamma$ agonists on human neoplastic cells in in vitro cultures of JAK2 ${ }^{\mathrm{V} 617 \mathrm{~F}}$ cells from human cell lines or primary $\mathrm{CD} 34^{+}$progenitors from $\mathrm{PV}$ and MF patients, with or without pioglitazone. First, we carried out a dose-response experiment and established that a concentration of $10 \mu \mathrm{M}$ pioglitazone was appropriate to decrease JAK2 ${ }^{\mathrm{V} 617 \mathrm{~F}}$ cells in culture (Figure 3, A and B). Under these conditions, pioglitazone treatment had a mild effect on the proliferation, survival, and clonogenicity of CD34+ progenitors from umbilical cord blood (Figure 3, C and $\mathrm{G})$ as previously reported $(20,21)$. This $\operatorname{PPAR} \gamma$ agonist-mediated defect of hematopoiesis in myeloid progenitors is not clinically significant in patients with otherwise normal hematopoiesis (37). Here, pioglitazone had a differential effect on JAK2 ${ }^{\mathrm{V} 617 \mathrm{~F}}$ cells, with a 4 -fold reduction in the number of living cells (at day 10) for the UKE-1 and HEL cell lines and a more than 3-fold reduction for $\mathrm{CD} 4^{+}$progenitors from $\mathrm{PV}$ and MF patients (Figure 3, A, B, D, and $\mathrm{E}$ ). We observed a similar effect in the clonogenic assay, with a significant inhibitory effect of pioglitazone of more than 2-fold in JAK2 ${ }^{\mathrm{V} 617 \mathrm{~F}}$ cells from the cell lines (Figure $3 \mathrm{~F}$ ) and cells from PV (Figure 3H) and MF patients (Figure 3I). 
A

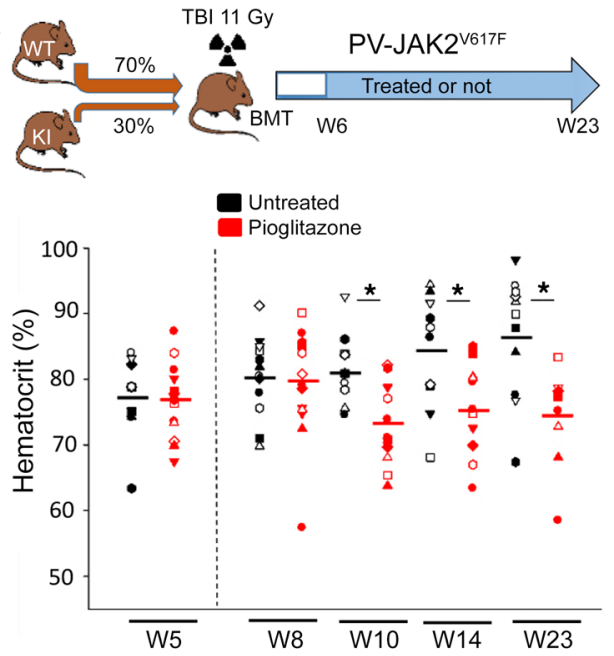

C

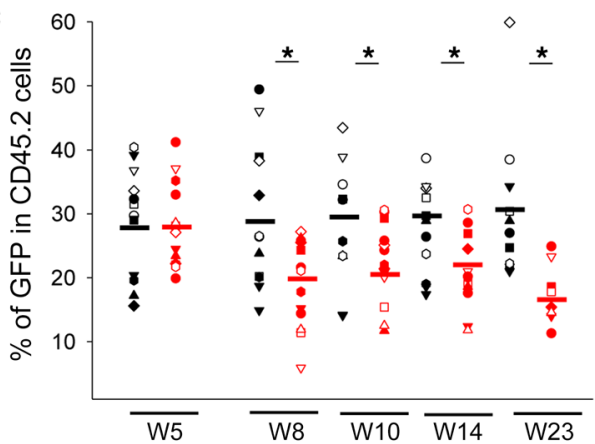

$\mathbf{E}$

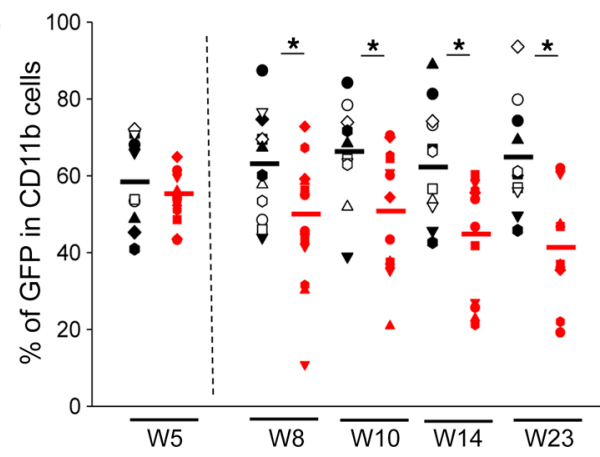

B
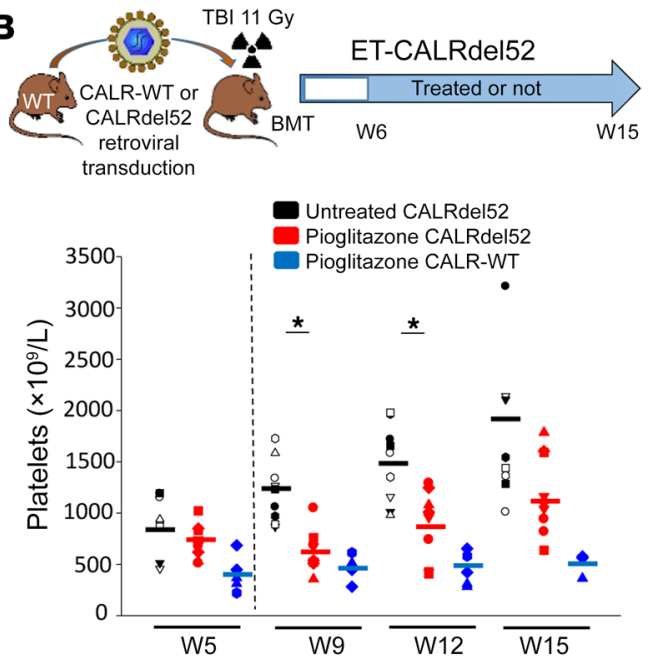

D

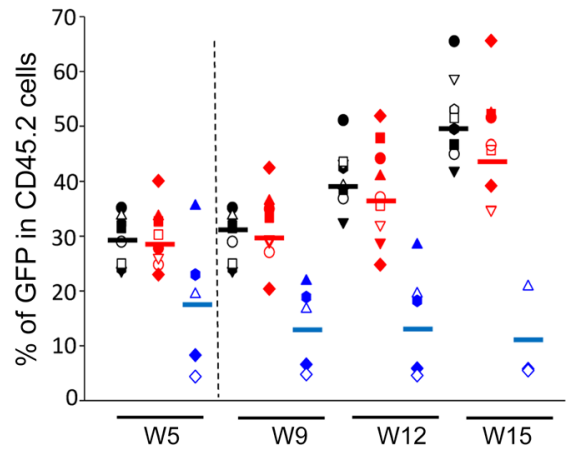

$\mathbf{F}$

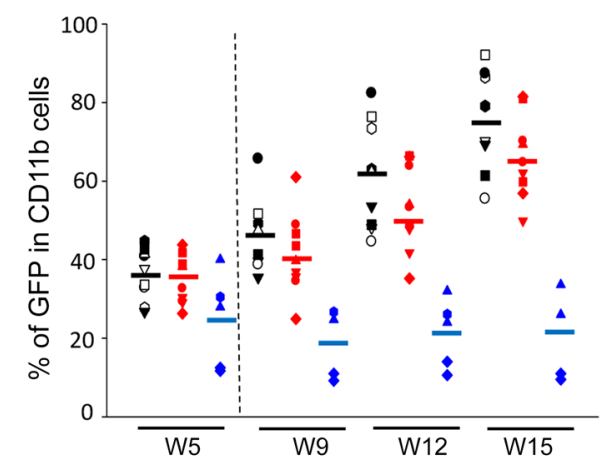

Figure 2. Treatment with PPAR agonists reduces myeloproliferation in PV-JAK2 $2^{\mathrm{V} 617 \mathrm{~F}}$ and ET-CALRdel52 mice. (A and B) Pioglitazone decreases the hematocrit of the PV-JAK2 2617F-GFP mice (A) and the platelet count of ET-CALRdel52 mice (B). (C and E) Pioglitazone reduces the proportion of the malignant JAK2 $2^{\mathrm{V} 617 \mathrm{~F}}$-GFP clone in the hematopoietic (CD45.2) cells of peripheral blood (C), especially in myeloid (CD11b) cells (E). (D and F) Pioglitazone limits expansion of the malignant CALRdel52-GFP clone in the hematopoietic (CD45.2) cells of peripheral blood (D), and the effect is greater in myeloid (CD11b) cells (F).

The black vertical dashed lines indicate initiation of treatment. The horizontal lines represent the mean. *Statistically significant difference $(P<0.05)$. ( $n=10$ mice per condition, except CALR-WT pioglitazone $n=5$.)
We then investigated the possible mechanisms that mediate pioglitazone activity against the JAK2 ${ }^{\mathrm{V} 617 \mathrm{~F}}$ cell lines. Apoptosis increased more than 3-fold (Supplemental Figure 4, A and A') and 5-ethynyl-2'-deoxyuridine (EdU) incorporation largely decreased in the presence of pioglitazone (Supplemental Figure 4, B and B'). This reduction in proliferation correlated with an increase in the proportion of cells in the $G_{0} / G_{1}$ stage of the cell cycle (Supplemental Figure 4, C and $C^{\prime}$ ). These results strongly suggest that pioglitazone promotes apoptosis and decreases proliferation of JAK2 $2^{\mathrm{V} 617 \mathrm{~F}}$ cells. We previously reported that PPAR $\gamma$ agonists negatively regulate the STAT5 pathway in hematopoietic progenitor cells (20-22), an effect that could be critical for the development of the JAK2 ${ }^{\mathrm{V} 617 \mathrm{~F}}$-driven PV model (38). To decipher whether the anti-myeloproliferative effect of pioglitazone could be partly explained by the downregulation of STAT5, we quantified STAT5 mRNA levels in UKE-1 and HEL JAK2 ${ }^{\mathrm{V} 617 \mathrm{~F}}$ cell lines treated or not with pioglitazone. We observed that pioglitazone treatment induced a significant reduction in STAT5A and STAT5B mRNA levels (Supplemental Figure 4, D and $\mathrm{D}^{\prime}$ ) and a decrease in STAT5 activation as shown by the reduction in phosphorylated STAT5 (p-STAT5; Supplemental Figure 4, E and $\mathrm{E}^{\prime}$ ).

Overall, these results highlight a PPAR $\gamma$-related anti-myeloproliferative effect on the neoplastic clone.

$P P A R \gamma$ activation in the resolution of MPN-related inflammation. MPNs represent a unique model of the relationship between the clonal development of a hematological malignancy and chronic inflammation. The PPAR $\gamma$ agonists (pioglitazone, mesalazine) potently reduced the white blood cell (WBC) count in the mouse models, both in the PV or ET stage and in MF (MF TPO ${ }^{\text {high }}$, post$\mathrm{PV}$, or post-ET; Figure $4 \mathrm{~A}$ ). In the aggressive murine model MF- 
A

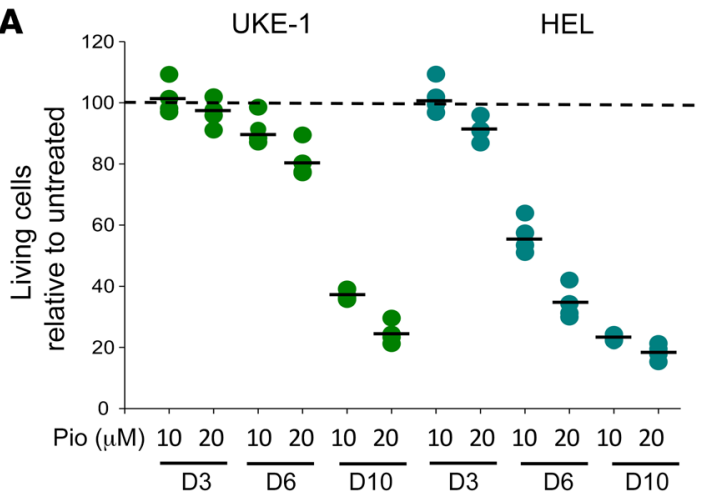

C

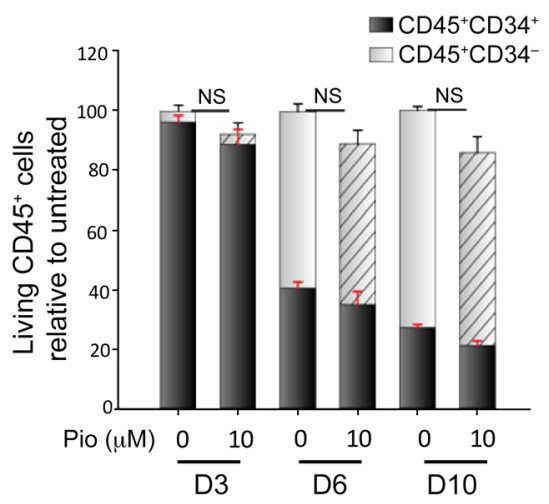

D

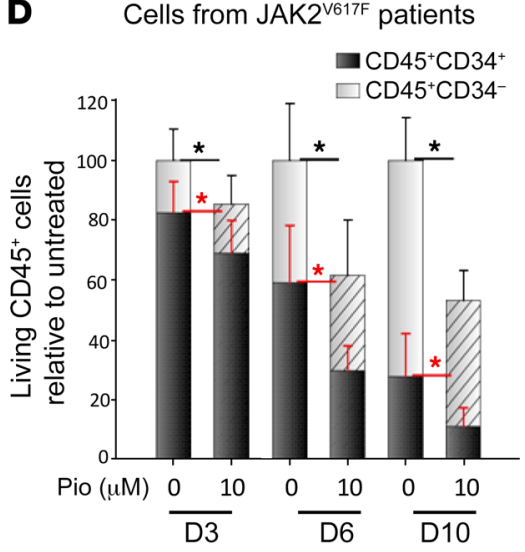

Cells derived from JAK2 $2^{\mathrm{V} 617 F}$ patient

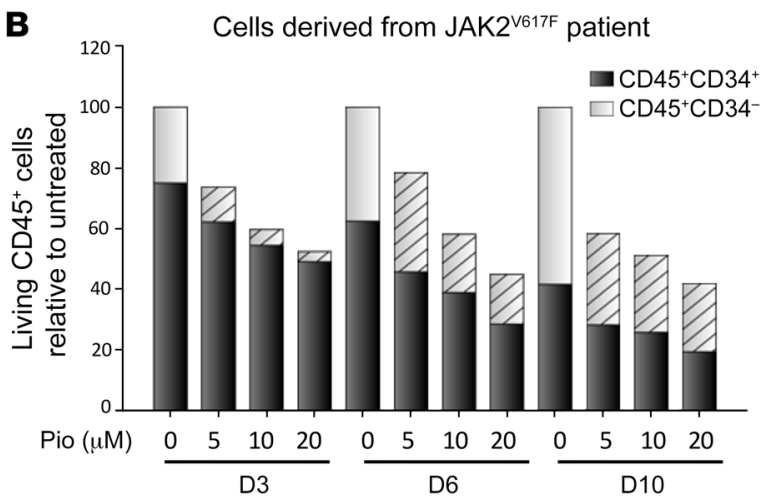

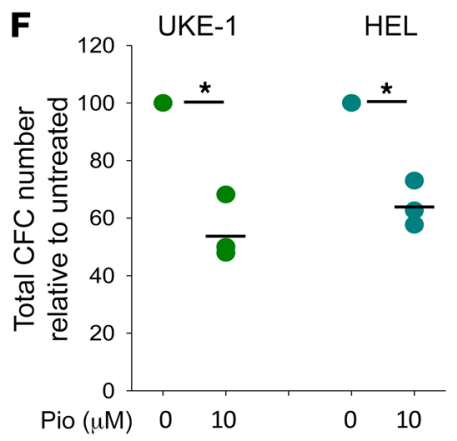
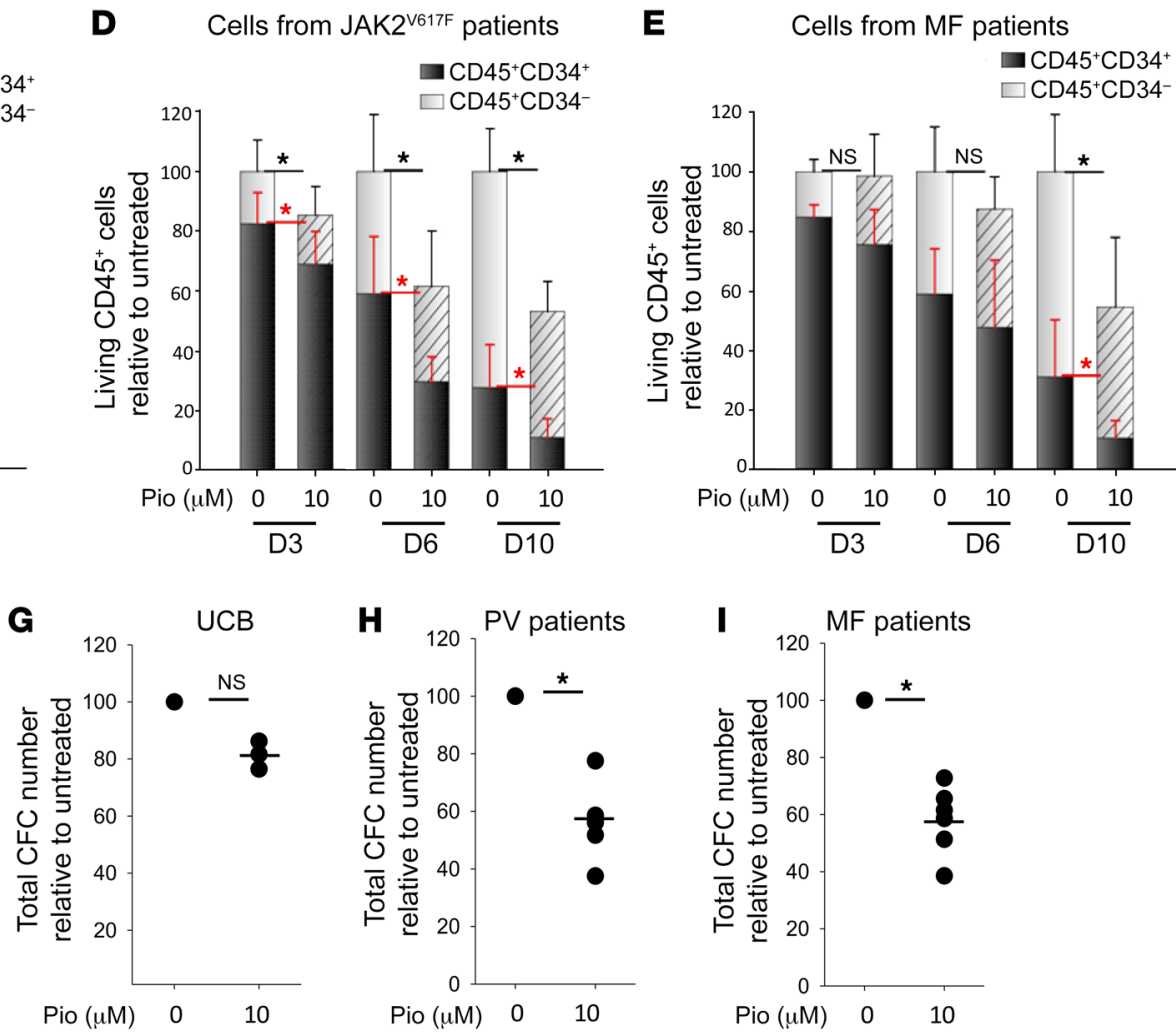

Figure 3. Antiproliferative effect of pioglitazone on human JAK2 ${ }^{\mathrm{V} 617 F}$ cell lines and hematopoietic progenitors from PV and MF patients. (A and B) Pioglitazone reduces the number of living cells in UKE-1 and HEL cell lines $(n=4)(\mathbf{A})$ and hematopoietic progenitors from a PV patient $(n=1)(B)$ in a dosedependent manner. (C) Pioglitazone slightly reduces the number of living umbilical cord blood (UCB) cells $(n=3)$. ( $\mathbf{D}$ and $\mathbf{E})$ Pioglitazone significantly reduces the number of living cells in hematopoietic progenitors from PV patients $(n=6)(\mathbf{D})$ and hematopoietic progenitors from MF patients $(n=8)(\mathbf{E})$. (F-I) Pioglitazone reduces the clonogenic potential of UKE-1 and HEL cells $(n=3)(\mathbf{F})$, hematopoietic progenitors from UCB (C), hematopoietic progenitors from PV patients $(n=5)(\mathbf{H})$, and hematopoietic progenitors from MF patients $(n=6)(\mathbf{I})$. The black horizontal lines represent the mean. ${ }^{*}$ Statistically significant difference $(P<0.05)$. CFC, colony-forming cell; $\mathrm{D}$, day.

TPO high, treatment dramatically reduced WBC count, but failed to completely normalize leukocytosis. Nevertheless, pioglitazone (and mesalazine to a lesser extent) counteracted MF-JAK2 ${ }^{\mathrm{V} 617 \mathrm{~F}_{-}}$ related leukocytosis and controlled the CALRdel52-related WBC increase. This regulation of WBC number affected both myeloid and lymphoid compartments, suggesting an effect on myeloproliferation and inflammation (Supplemental Figure 5, A-D).

We then investigated the possible players that mediate this $\operatorname{PPAR} \gamma$ antiinflammatory activity in JAK2 ${ }^{\mathrm{V} 617 \mathrm{~F}}$ mice, treated or not with pioglitazone (at $\mathrm{W} 6$ after transplantation), by assessing mRNA levels of 9 putative inflammatory mediators (39) in nucle- ated blood cells (W8, W14, and W23 after transplantation). Perilipin-2, known to be upregulated by PPAR $\gamma$ agonists in vivo (40), was used as a positive control and confirmed the progressive uptake of pioglitazone by blood cells (Figure 4B). There was no difference in the mRNA levels of IL-15, IFN- $\gamma$, IL-1 $1 \beta$, or IL-12 (p40) between treated and untreated mice. There was a trend toward a reduction of $T n f-\alpha$ and $I L-5$ gene expression. Seventeen weeks after the initiation of pioglitazone treatment (W23 after transplantation), TGF- $\beta$ mRNA levels were significantly lower in treated than in untreated mice, and there was a concomitant significant

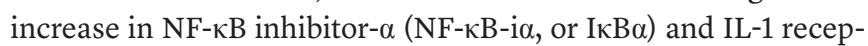


tor antagonist (IL-1ra) mRNA levels (Figure 4B). Il-1ra belongs to the IL-1 family and binds to IL-1 receptors without inducing a cellular response, thereby behaving as a decoy receptor for IL-1 $\alpha$ and IL- $1 \beta$ (41). Because TGF- $\beta$ is a key factor in MF, we assayed plasma TGF- $\beta$ in the TPO ${ }^{\text {high }}$ model (W8) and confirmed that treatment with PPAR $\gamma$ ligands (pioglitazone or mesalazine) significantly reduced the TGF- $\beta$ level (Supplemental Figure $5 \mathrm{E}$ ). In addition, JAK2 ${ }^{\mathrm{V} 617 \mathrm{~F}}$ mice treated with PPAR $\gamma$ agonists had significantly smaller spleens (Table 1), which suggests better control of MF-related inflammation. Overall, these results support an antiinflammatory role of PPAR $\gamma$ in MF.

PPAR $\gamma$ activation abrogates TGF- $\beta 1$ signaling by trapping the p300 cofactor in BM stromal cells. The fibrotic process induced by TGF- $\beta 1$ is driven by a combination of increased matrix biosynthesis, including collagen (types I, III, and IV), and the deposition of fibronectin, accompanied by a decrease in matrix degradation (particularly by an increase in the tissue inhibitor of metalloproteinases TIMP-1) (28). Importantly, certain TGF- $\beta$ related profibrotic effects are mediated through upregulation of its downstream effector, connective tissue growth factor (CTGF). Like collagen and fibronectin, CTGF is induced by the canonical TGF- $\beta /$ Smad pathway, stimulates fibroblast differentiation and collagen synthesis (42), and potentiates TGF- $\beta$ mediated fibrogenesis (43).

Stimulation by TGF- $\beta 1$ induced an increase in CTGF mRNA levels in murine (MS5) and human (HS5) BM stromal cell lines and human primary BM stromal cells of 70-, 9-, and 3-fold, respectively (Figure 5, A-C). This effect was dependent on TGF- $\beta$ type 1 receptor (Alk5), as it was abrogated by a specific inhibitor of activin receptor-like kinase (Alk) (SB-431542). The addition of pioglitazone significantly counteracted the TGF- $\beta 1$-related induction of CTGF, with a reduction of $67 \%, 75 \%$, and $58 \%$ in MS5, HS5, and primary human BM stromal cells, respectively (Figure 5, A-C). We also obtained similar results for other TGF- $\beta 1$ target genes: collagen type I $\alpha 1$ (Col1a1) and fibronectin (Supplemental Figure $6, A-D)$. This inhibitory effect of PPAR $\gamma$ agonists on TGF- $\beta 1$ transcriptional activity was not reciprocal, as expression of the PPAR $\gamma$ target gene perilipin-2 (PLIN-2) was not affected by the presence of TGF- $\beta 1$ (Supplemental Figure 6, E and F).

We analyzed the canonical TGF- $\beta /$ Smad signaling pathway to decipher the molecular mechanisms that govern interaction between PPAR $\gamma$ and TGF- $\beta$ pathways. Upon phosphorylation by the activated type 1 TGF- $\beta$ receptor, cytoplasmic Smad 2 and Smad3 heterodimerize with Smad4 and accumulate in the nucleus, where they recruit cofactors to genomic Smad-binding elements. The addition of pioglitazone did not affect the phosphorylation of Smad2/3 induced by TGF- $\beta 1$ stimulation (Supplemental Figure $6, \mathrm{G}$ and $\mathrm{H}$ ), suggesting that the initial steps of the TGF- $\beta$ pathway activation remain unaltered. Several studies have reported that activation of PPAR $\gamma$ negatively regulates canonical TGF- $\beta$ signaling in skin fibroblast and lung smooth muscle cells, probably by competing with Smad proteins for the recruitment of the p300 acetyltransferase cofactor to the Smad-responsive elements (44, 45). We hypothesized that a similar mechanism may be present in stromal BM cells (Figure 6A). We first assessed the effect of an inhibitor of histone acetyltransferase (C646) that is differentially selective for p300 versus other acetyltransferases. C646 disrupt- ed TGF- $\beta$ signaling by reducing the increase of CTGF mRNA levels by $40 \%$ and $50 \%$ in the MS5 and HS5 cell lines, respectively, and abrogated the repressive effect of pioglitazone on TGF- $\beta$ signal transduction (Figure 5, D and E). Under the same conditions, the MEK1/2 inhibitor U0126 and the JNK1-3 inhibitor SP600125, which target TGF- 31 -dependent non-Smad-signaling MKK6-p38, MEK-ERK, and JNK/MAPK, had no effect (data not shown), suggesting that the repressive effect of pioglitazone is mainly mediated by the Smad/histone acetyltransferase p300 pathway. We overexpressed WT p300 (p300 HAT) or a mutant form of p300 lacking functional histone acetyltransferase activity (p300 HAT-) or the empty GFP lentiviral vector in MS5 BM stromal cells to clarify the role of $\mathrm{p} 300$. In comparison with control GFP lentiviral vector, in MS5 cells stimulated by TGF- $\beta 1$, p300 HAT ${ }^{-}$overexpression had no effect on the repressive activity of pioglitazone, whereas functional p300 HAT abrogated the repressive effect related to PPAR $\gamma$ activation (Figure 5, F-I).

We hypothesized that activation of PPAR $\gamma$ could repress the TGF- $\beta$-dependent transcriptional induction of fibrosis genes by trapping p300 in BM stromal cells (Figure 6A). We thus performed ChIP-Seq analysis using a specific antibody directed against p300 in the MS5 BM stromal cell line stimulated with TGF- $\beta 1$, with or without pioglitazone. We also performed parallel ChIP-Seq experiments with a c-Jun-specific antibody, because AP-1 sites and their cognate transcription factors also play important roles in the gene regulatory activities of TGF- $\beta$. We compared the genome-wide localization of $\mathrm{p} 300 \mathrm{ChIP}$-Seq peaks between various conditions (i.e., TGF- $\beta 1$ vs. TGF- $\beta 1+$ pioglitazone) and observed 3 clusters (Figure 6B), which we called clusters I, II, and III. Cluster II was composed of 16,077 common peaks, representing sites that were similarly occupied by $\mathrm{p} 300$ in both the TGF- $\beta 1$-treated and the TGF- $\beta 1$ + pioglitazone-treated cells. Conversely, clusters I and III, which consisted of 15,389 and 5917 peaks, respectively, represent p300 binding sites showing dynamic occupancy upon pioglitazone addition. The sites of cluster I showed a large decrease in p300 occupancy in the presence of pioglitazone, whereas those of cluster III showed an opposite trend, with either increased or de novo occupancy in the presence of pioglitazone. These results strongly suggest that PPAR $\gamma$ activation relocalizes p300 sites to novel genomic locations, thus altering the TGF- $\beta 1$ regulatory landscape. This large reorganization of the genome-wide occupancy was specific for $\mathrm{p} 300$, as the c-Jun profiles remained unchanged upon pioglitazone addition (Figure 6B), suggesting that AP- 1 is not involved in the interactions between TGF- $\beta 1$ and PPAR $\gamma$ signaling. We then assigned putative target genes to the various p300 peaks using the nearest-gene approach. Comparison of the target genes in each condition showed a large decrease in TGF- $\beta 1$-associated genes, with the appearance of 125 target genes, upon pioglitazone addition (Figure 6C). This is consistent with substantially decreased p300 occupancy observed in the cluster I sites and de novo occupancy observed in cluster III (Figure 6B). These results demonstrate the ability of PPAR $\gamma$ activation to inhibit TGF- $\beta$ / Smad signal transduction by trapping of the p300 cofactor, thus likely preventing p300 recruitment by phosphorylated Smads to key genomic sites. We performed a Gene Ontology (GO) enrichment analysis of the 3 clusters to obtain functional information and confirmed a reduction in the regulation of TGF- $\beta$ production 
A
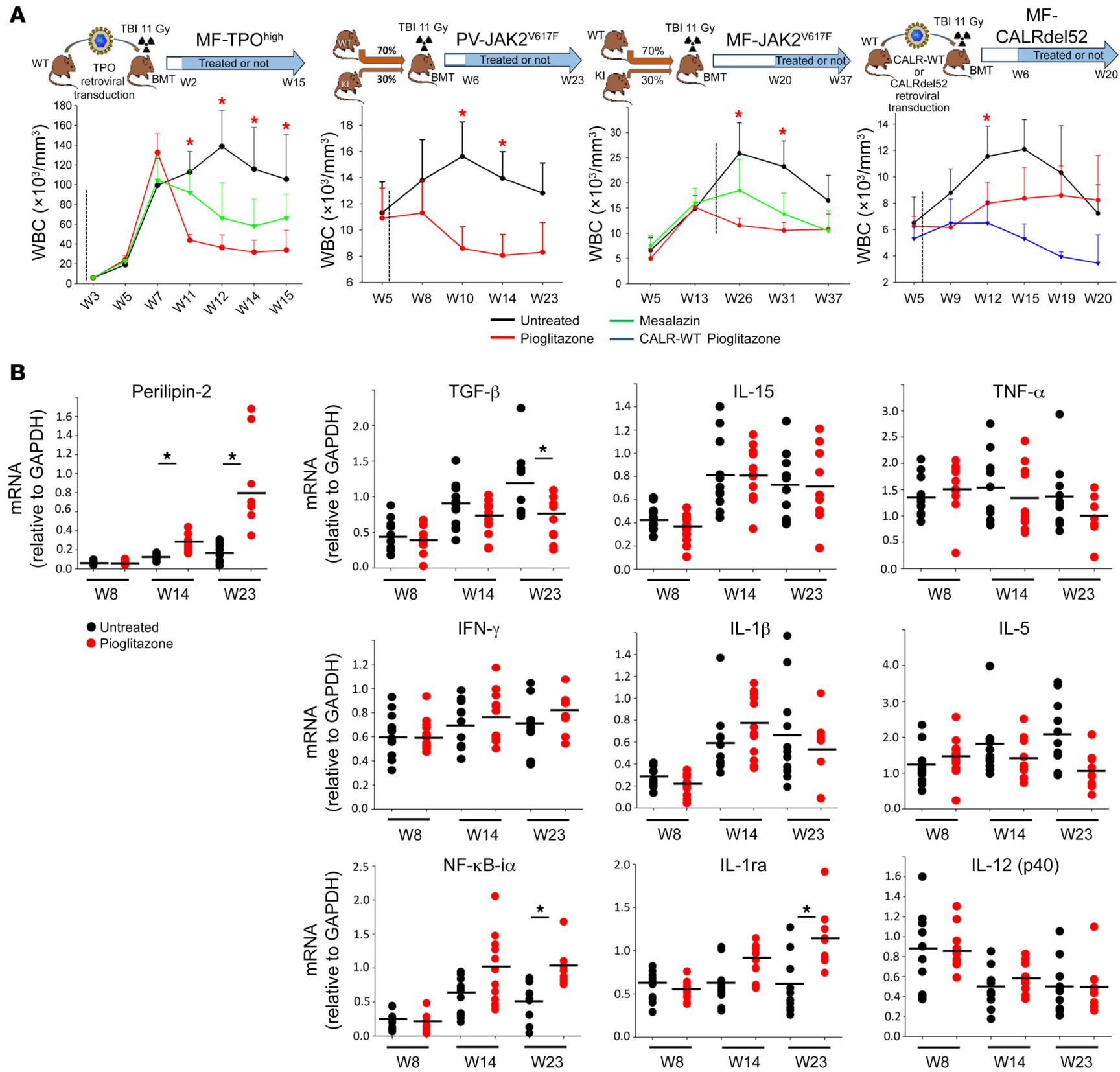

Figure 4. Resolution of inflammation with PPAR $\gamma$ agonists in murine models of MPN. (A) PPAR $\gamma$ agonists decrease leukocytosis related to the MPN phenotype. ( $n=10$ mice per condition, except CALR-WT pioglitazone $n=5$.) (B) Quantification of mRNA transcript levels of inflammation-related genes in nucleated cells from the peripheral blood shows an antiinflammatory profile in treated JAK2 ${ }^{\mathrm{V} 617 \mathrm{~F}}$ mice (red, $n=10$ ) relative to that in untreated JAK2 $2^{\mathrm{V} 617 \mathrm{~F}}$ mice (black, $n=10)$. The black horizontal lines represent the mean. ${ }^{*}$ Statistically significant difference $(P<0.05)$.

and the TGF- $\beta$ signaling pathways in the presence of pioglitazone (Supplemental Figure 7A). We next focused on MF, by analyzing the average enrichment of a panel of genes directly associated with fibrosis, and showed, by p300 chromatin immunoprecipitation (ChIP), that the addition of pioglitazone potently reduced the ability of TGF- $\beta$ to induce the transcription of fibrosis genes (Supplemental Figure 7B). We observed no enrichment by c-Jun ChIP (Supplemental Figure 7B). We also observed enrichment of a panel of inflammatory genes that was reduced in the presence of pioglitazone, shown by p300 immunoprecipitation (Supplemental Figure 7C). We did not detect any enrichment in a panel of genes involved in cell proliferation and survival under the same conditions (Supplemental Figure 7D). In order to compare ChIPSeq peak enrichment and gene expression, we targeted a panel of 14 genes related to MF whose mRNA level was modulated after induction by TGF- $\beta$ alone versus TGF- $\beta$ + pioglitazone (Supplemental Figure 8). Among them, the 9 genes involved in fibrosis development (Ctgf, Col1a2, Col5a3, Vim, Fn1, Krt7, Krt8, Acta2, Timp1), the master gene for osteosclerosis (Runx2), and the 2 proinflammatory genes $(N l r p 3, N f \kappa B 1)$ appeared to be repressed in the presence of pioglitazone (Supplemental Figure 8A) and to present a significant reduction of ChIP-Seq peak enrichment (Sup- 
A

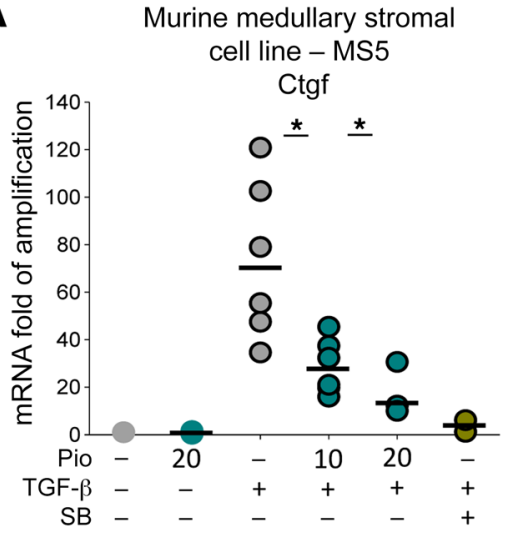

D

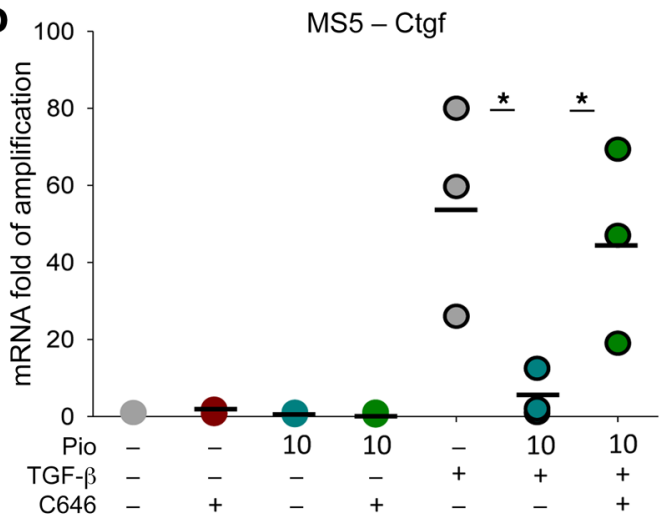

B

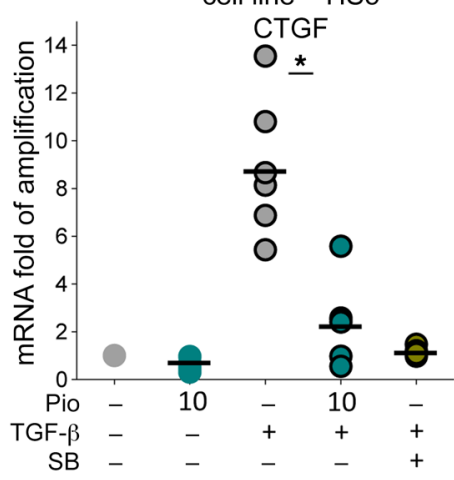

C Primary cells derived from

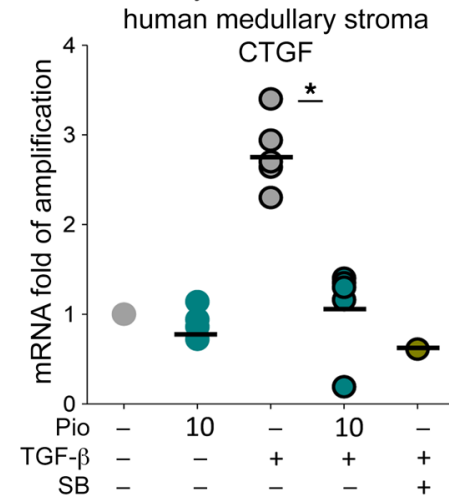

E

HS5 - CTGF
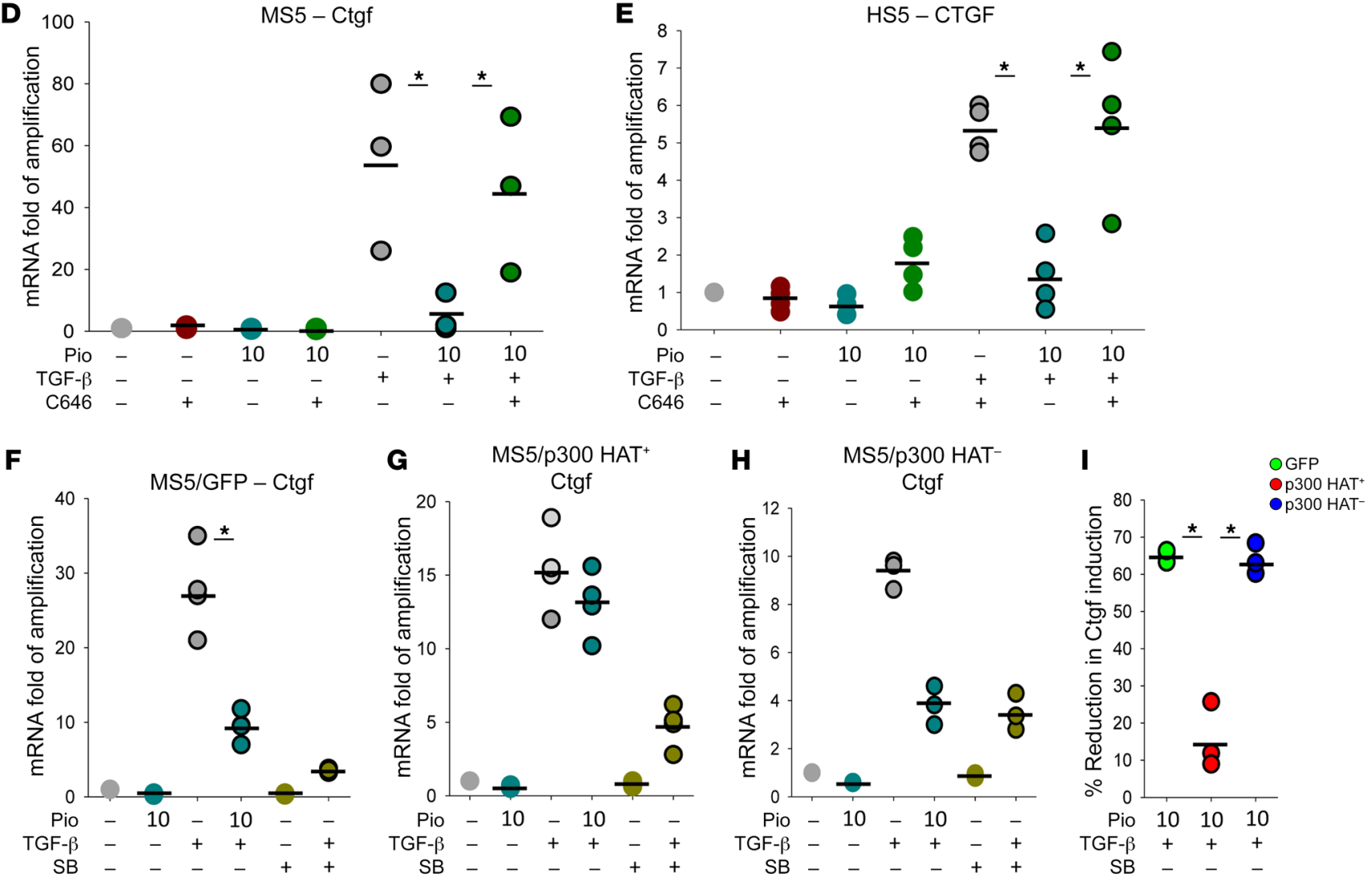

Figure 5. Pioglitazone reduces induction of the TGF- $\mathbf{\beta 1}$ profibrotic target gene CTGF in a p300-dependent manner. (A-C) Pioglitazone decreases the induction of transcription of the CTCF gene induced by TCF- $\beta 1$ in MS5 BM stromal cells (A), HS5 BM stromal cells (B), and primary BM stromal cells (C). ( $\mathbf{D}$ and $\mathbf{E}$ ) The inhibitory effect of pioglitazone is abrogated by the chemical inhibitor of the HAT activity of the p300 cofactor C646 in MS5 BM stromal cells (D) and HS5 BM stromal cells (E). (F-H) Overexpression of the efficient p300 protein (HAT+) in MS5 BM stromal cells abrogates the inhibitory effect of pioglitazone on the induction of CTGF related to TGF- $\beta 1$ stimulation (C) relative to control transduction with an empty lentivirus (F), whereas overexpression of the deficient $\mathrm{p} 300$ protein (HAT-; $\mathbf{H}$ ) has no effect (graphs are representative of 1 experiment). (I) Percentage of reduction in cumulative analysis $(n=4)$. The black horizontal lines represent the mean. ${ }^{*}$ Statistically significant difference $(P<0.05)$. CTCF, connective tissue growth factor; HAT, histone acetyltransferase; SB, SB-431542 (inhibitor of TGF- $\beta 1$ receptor).

plemental Figure 8C). The NfאBia and Gas1 genes, which were not repressed in the presence of pioglitazone (Supplemental Figure 8B), did not exhibit any reduction in ChIP-Seq peak enrichment (Supplemental Figure 8D).

These data argue that PPAR $\gamma$ agonists play a role in limiting fibrosis, mainly via inhibition of TGF- $\beta /$ Smad signal transduction by trapping of the cofactor $\mathrm{p} 300$.

\section{Discussion}

Cumulative evidence indicates that the survival and development of hematological neoplasms are dependent not only on the cell-intrinsic functional properties of the driver mutations present within the cancer stem cell, but also on the ability of surrounding cells to support the malignant clone. This observation is particularly relevant to MPNs, especially in MF, which is the prototypical example 


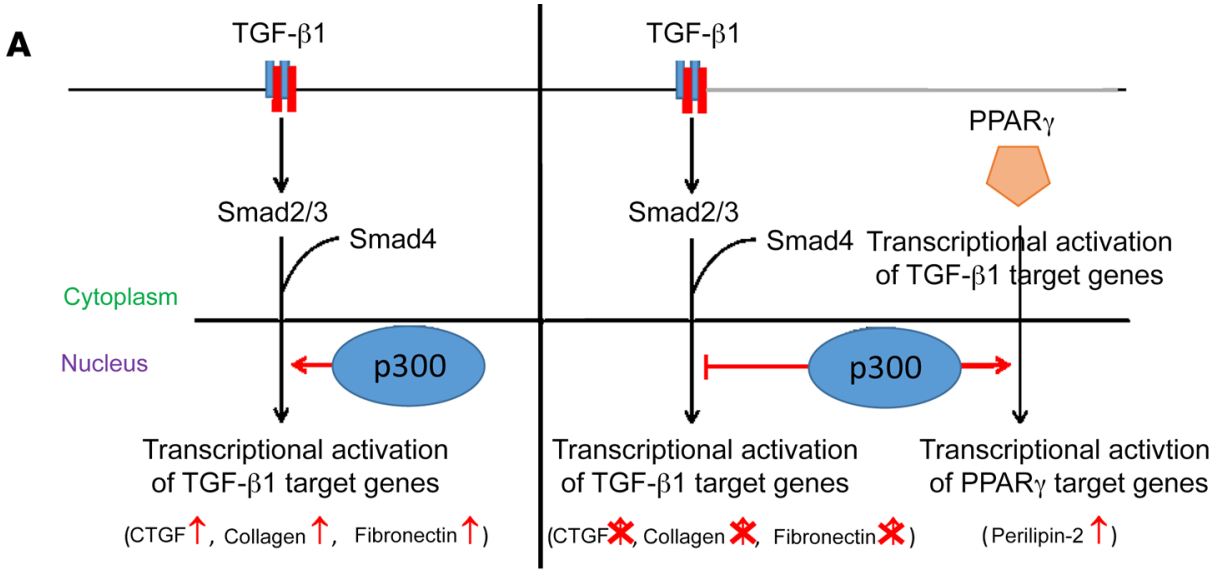

B

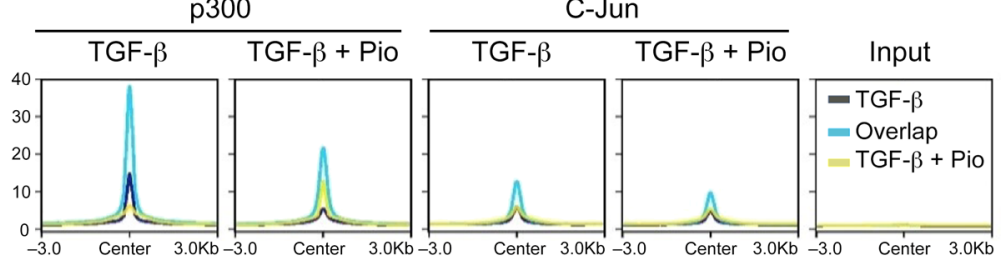

C

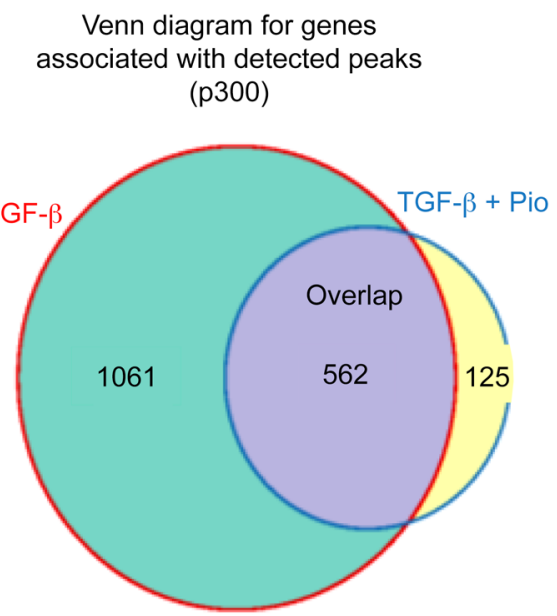

Figure 6. PPAR $\gamma$ activation counteracts the induction of TGF- $\beta 1$ target genes by sequestering the $\mathbf{p} 300$ transcription cofactor. (A) Schematic representation of the mechanistic hypothesis. Activation of the PPAR $\gamma$ nuclear receptor by its ligand (pioglitazone) leads to the recruitment of the p300 cofactor for the transcription of PPAR $\gamma$ target genes to the detriment of the transcription of TCF- $\beta 1$ target genes. (B) Heatmap representation of the genomewide analysis. Left: The 3 clusters of genes I, II, and III. I (TCF- $\beta$ ) = target genes of TCF- $\beta 1$; III (pioglitazone) = target genes of PPAR $\gamma$; II (overlap) = common genes. Top: Stimulation condition (TGF- $\beta$ alone or TGF- $\beta+$ pioglitazone) and antibody target for immunoprecipitation (p300 or c-Jun). Bottom: Center gene distance. Cluster I sites show a large decrease of p300 occupancy in the presence of pioglitazone, whereas cluster III sites show the opposite trend, with increased or de novo occupancy in the presence of pioglitazone. (C) Venn diagram for target genes associated with detected peaks ( $\mathrm{p300}$ ). The presence of pioglitazone led to a decrease in the number of TCF- $\beta 1$ genes and the appearance of 125 target genes related to PPAR $\gamma$. TES, transcription end site; TSS, transcription start site. of interactions between a clonal disorder arising in the hematopoietic stem cell and proinflammatory cytokines that activate stromal cells in the BM, support their fibrotic activity, and reduce their capacity to support hematopoiesis. MF is a life-threatening disease with significantly shortened survival (4). Despite our advanced understanding of the molecular pathogenesis of MPNs, currently available treatments are mainly symptomatic. JAK inhibitor monotherapy has little effect on BM fibrosis (9-11). Nevertheless, slowing or reversing BM fibrosis is a clinically achievable goal as demonstrated in MF patients after allogenic stem cell transplanta- 
tion $(46,47)$. The potential therapeutic approaches for MF can be divided into 3 aims: (a) to restrict the expansion of the malignant clone, (b) to dampen inflammation, and (c) to target BM fibrosis. Here, we provide evidence using 3 different mouse models of MF to support a therapeutic potential of PPAR $\gamma$ agonists for clonogenicity, inflammation, and fibrosis.

We previously reported that PPAR $\gamma$ agonists differentially repress normal and pathological hematopoiesis (20-22). Recently the invalidation of PPAR $\gamma$ signaling has been associated with the expansion of human hematopoietic stem and progenitor cells $(48,49)$. Here, we show that pioglitazone can reduce the myeloproliferative state by significantly reducing the hematocrit and platelet level in preclinical murine models of PV (JAK2 $\left.{ }^{\mathrm{V} 617 \mathrm{~F}}\right)$ and ET (CALRdel52). Jointly, we observed a reduction in the density of MKs in BM from the JAK2 ${ }^{\mathrm{V} 617 \mathrm{~F}}$ and CALRdel52 models and a normalization of MK size in the CALRdel52 model, suggesting an effect on MK hyperplasia. In the mouse model of PV, this effect was associated with a decrease in the proportion of JAK2 ${ }^{\mathrm{V} 617 \mathrm{~F}}-\mathrm{GFP}$ cells, in both the mature hematopoietic cell and the hematopoietic stem and progenitor cell (LSK) compartments. Nonetheless, this effect appears to be restricted to multipotential progenitors and cells with a short-term capacity of repopulation (LSK-CD34 ${ }^{+}$). In the mouse model of ET, the reduction of platelet counts was not associated with a significant reduction in the proportion of CALRdel52-GFP cells, suggesting that pioglitazone activity is not limited to the mutated CALRdel52 cells. This result can be partially explained by activation of the CALRdel52-negative cells through a paracrine mechanism mediated by mutated CALR (50) that could induce targeting of these CALRdel52-negative cells and reduce specificity on CALRdel52-GFP cells. Alternatively, some of the effects of CALRdel52 may be mediated not by the MPL/JAK2/ STAT pathway but by induction of ER stress and/or modification of $\mathrm{Ca}^{2+}$ signaling. We showed that pioglitazone is more effective in mutated JAK2 ${ }^{\mathrm{V} 617 \mathrm{~F}}$ hematopoietic progenitor cells than in normal hematopoietic progenitors in vitro and ex vivo. This repressive effect changed both the clonogenic and the proliferative potential of the JAK2 $2^{\mathrm{V} 617 \mathrm{~F}}$ cells and was associated with an increase in the proportion of apoptotic cells and cells in the $G_{0} / G_{1}$ stage of the cell cycle. In MPNs, the 3 driver mutations, JAK2 ${ }^{\mathrm{V} 617 \mathrm{~F}}$ ( $\sim 70 \%$ of MPNs) and mutated CALR and MPL ( $20 \%$ of MPNs), and a large proportion of the remaining MPNs called triple-negative are associated with increased JAK2/STAT5 signaling (51). As we have previously reported (20-22), we observed here that pioglitazone treatment reduces STAT5 signaling. The downregulation of STAT5 activity by PPAR $\gamma$ agonists may partially explain the anti-myeloproliferative effect of pioglitazone and mesalazine. Indeed, STAT5 is absolutely required for the pathogenesis of PV in the JAK2 ${ }^{\mathrm{V} 617 \mathrm{~F}}$ mouse model (38). PPAR $\gamma$ agonists may also act by counteracting the Wnt/ $\beta$-catenin pathway, which is involved in hematopoietic cell proliferation and aberrantly activated in MPNs $(52,53)$. Further studies are needed to decipher the mechanisms by which the activation of PPAR $\gamma$ affects myeloproliferation in MPNs.

Chronic inflammation is a driver of clonal evolution in MPN (54). It is responsible for debilitating symptoms, and there is growing evidence suggesting an association between leukocytosis and thrombosis, in both PV and ET (55). In the clinic, leukocytosis is frequently found in PV or early MF, and a small increase in WBC counts is commonly described in ET (56). Similar observations have been reported in preclinical mouse models of these diseases $(15,16,18)$. The neoplastic clone appears to be the main driver of the inflammatory microenvironment of MPNs with the continuous release of inflammatory molecules from activated leukocytes and platelets (57). It has been shown in animal models that cells of both innate and adaptive immunity are involved in the pathogenesis of MPN, suggesting that the observed strong inflammation is due not only to the MPN clone, but also to an inflammatory reaction of nonmalignant cells (58). Given the importance of inflammatory cytokines in the emergence and evolution of MPNs (54) and fibrotic BM niche remodeling in mouse models (59), it is crucial to dampen inflammation in MF. PPAR $\gamma$ activation has long been studied for its antiinflammatory properties, and certain agonists (for example, mesalazine) have been used for over 30 years in the treatment of chronic inflammatory diseases, including inflammatory bowel diseases (60) and rheumatoid arthritis (61). The resolution of inflammation is an active and dynamic process that represents not only an increase in antiinflammatory actions, but also a shift in immune cell functions to restore homeostasis. PPAR $\gamma$ can shift production by neutrophils, platelets, macrophages (62), and MKs (26) from pro- to antiinflammatory mediators. Among the inflammatory cytokines associated with the development of MF, pioglitazone has been shown to extensively and specifically affect the expression of TNF- $\alpha$ in a wide range of inflammatory models, including sepsis, ischemia/reperfusion, colitis, and spinal trauma models (63-65). Additionally, PPAR $\gamma$ and TGF- $\beta$ repress each other's expression in fibroblasts $(66,67)$. Despite great interest, therapies targeting TGF- $\beta$ alone have so far shown disappointing outcomes in clinical trials (68). Here we showed that targeting PPAR $\gamma$ to decrease TGF- $\beta$ expression could be an interesting alternative. Transcriptional control of the vast majority of genes involved in inflammation requires $\mathrm{NF}-\kappa \mathrm{B}$ activation, and the broad-ranging effects of PPAR $\gamma$ agonists on proinflammatory cytokines may be mediated by the regulation of NF- $\kappa \mathrm{B}$ activation. PPAR $\gamma$ could repress NF- $\kappa \mathrm{B}$ signaling by physically interacting with $\mathrm{NF}-\kappa \mathrm{B} / \mathrm{p} 65$ and inducing its degradation (69), or by upregulating $\mathrm{I} \kappa \mathrm{B} \alpha$, a negative regulator of $\mathrm{NF}-\kappa \mathrm{B}(70)$. By masking the NF- $\kappa \mathrm{B}$ nuclear localization signal, the inhibitory protein NF- $\kappa \mathrm{B}-\mathrm{i} \alpha$ sequesters NF- $\kappa \mathrm{B}$ as an inactive complex in the cytoplasm (71). Pioglitazone treatment induced a significant decrease in TGF- $\beta$ mRNA levels and a trend toward decreased TNF- $\alpha$ mRNA levels, associated with a significant increase in I $\mathrm{KB} \alpha$ and IL-1ra gene expression in nucleated blood cells in the MF post-PV JAK2 ${ }^{\mathrm{V} 617 \mathrm{~F}}$ model. IL-1ra competitively antagonizes the inflammatory effects of IL-1 $\alpha$ and IL-1 (41), and its expression has also been reported to be regulated by pioglitazone in ischemic brain (72). Neutrophils are the first responders to most inflammatory stimuli, and neutrophilia is a hallmark of many inflammatory diseases. The effect of pioglitazone on neutrophil function and counts has been summarized by Croasdell et al. (62). PPAR $\gamma$ activation has been shown to decrease neutrophil numbers and myeloperoxidase activity in various models of inflammation (lipopolysaccharide challenges, high-fat diet, ischemia/reperfusion, colitis, and gastric injury) (62). In our mouse models of MPN, we demonstrate that PPAR $\gamma$ agonists (pioglitazone and mesalazine) can potently reduce the WBC count. We also show that PPAR $\gamma$ agonists help to reduce spleen size. Overall, these observations argue for an antiinflammatory role of PPAR $\gamma$ in MF. 
Prevention or limitation of the development of BM fibrosis could help to restore normal hematopoiesis and disrupt the malignant self-reinforcing niche. PPAR $\gamma$ agonists have shown antifibrotic effects in many organs, including the lungs, skin, kidneys, eyes, heart, liver, and bowel (73). Fibrosis is associated with reduced tissue levels of PPAR $\gamma$ in certain organ-specific human fibrotic diseases (kidney, liver, lung, and the cutaneous lesions of scarring [cicatricial] alopecia) (74) and in various mouse models of fibrotic diseases (75). Furthermore, genetic deletion of PPAR $\gamma$ in the liver (76) and mouse embryonic fibroblasts (MEFs) (77) exacerbates fibrogenesis after organ injury. PPAR $\gamma$-null MEFs show evidence of autocrine TGF- $\beta$ stimulation with enhanced TGF- $\beta$ production, constitutive activation of Smad2/3, and upregulation of collagen synthesis, whereas reconstitution of these cells with ectopic PPAR $\gamma$ resulted in their normalization (77). Here we demonstrate, for the first time to our knowledge, that profibrotic signaling triggered by TGF- $\beta 1$ is counteracted by PPAR $\gamma$ agonists in murine and human BM stromal cell lines and in primary BM stromal cells from patients. The mechanisms reported to be involved in blockage of the canonical Smad signaling pathway by PPAR $\gamma$ activation appear to be tissue dependent, with reduced Smad3 phosphorylation in hepatic stellate cells (78) and involvement of the p300 cofactor in fibroblasts (45). We demonstrated that the presence of pioglitazone does not prevent Smad2/3 phosphorylation in BM stromal cells but counteracts TGF- $\beta$ profibrotic signaling in a p300-dependent manner. We observed a competitive shift of the p300 cofactor from TGF- $\beta$ transcriptional signaling to PPAR $\gamma$ transcriptional signaling in the presence of pioglitazone by ChIPSeq analysis. Such trapping of p300 by activated PPAR $\gamma$ potently represses the ability of TGF- $\beta$ to induce profibrotic genes. Moreover, PPAR $\gamma$ activation may also restrict osteosclerosis associated with MF (4), as observed in the histopathological analysis of BM from the MF post-JAK2 ${ }^{\mathrm{V} 617 \mathrm{~F}}$ mouse models. Indeed, there is transcriptional antagonism between Runt-related transcription factor 2 (RUNX2) and $\operatorname{PPAR} \gamma$, the two key regulators of mesenchymal differentiation, to orient differentiation toward osteoblasts or adipocytes, respectively (79), and activation of PPAR $\gamma$ promotes adipocyte differentiation to the detriment of osteoblast formation.

Our results show that PPAR $\gamma$ agonists may be effective on the 3 components of MF pathophysiology. As a final demonstration, we showed that PPAR $\gamma$ agonists reduce the development of both reticulin fibers and osteosclerosis in $\mathrm{BM}$ and contribute to reduction of spleen size in the JAK2 ${ }^{\mathrm{V} 617 \mathrm{~F}}$ mouse model. We anticipate that the addition of PPAR $\gamma$ agonists to anti-JAK2 therapy, such as ruxolitinib, may have synergistic effects on both constitutional symptoms and BM fibrosis in MF patients. Further clinical trials in MF patients may help to determine the best way to use PPAR $\gamma$ agonists in clinical practice.

\section{Methods}

\section{Mice}

The JAK2 ${ }^{\mathrm{V} 617 \mathrm{~F}}$-knockin (KI) mice have been previously described (15). Our PV and MF post-PV models were generated by transplantation of a mixture of JAK2 ${ }^{\mathrm{V} 617 \mathrm{~F}}$-KI (Ly5.2; one-third) and WT (Ly5.1; twothirds) BM cells into lethally irradiated recipients ( $11 \mathrm{~Gy}$ ) as previously described (15). The CALRdel52 and CALR-WT retroviral murine models have been previously described (16), and were used to generate ET and MF post-ET. Briefly, 4 days after 5-fluorouracil (5-FU) treatment (150 mg/kg), BM cells from WT C57BL/6 mice (Charles River Laboratories, France) were cocultivated for 4 days with pMSCV-CALRwtIRES-GFP or pMSCV-CALRdel52-IRES-GFP virus-producing Plate-E cells in DMEM containing IL-3, SCF, TPO, and 20\% FCS and transplanted into lethally irradiated recipients. The previously described $\mathrm{TPO}^{\text {high }}$ mice (17) were used to generate the MF-TPO ${ }^{\text {high }}$ model. Briefly, 5-FU-treated BM cells from WT C57BL/6 mice were cocultivated for 3 days with MPZenTPO virus-producing GP/E-86 cells in DMEM containing IL-3, SCF, and TPO. After 3 days nonadherent cells were collected and transplanted into lethally irradiated recipients.

\section{Patient samples and cell lines}

Information about PV and MF patients is presented in Supplemental Tables 1 and 2, respectively.

CD $34^{+}$hematopoietic progenitors. CD34 ${ }^{+}$cells from patients with PV or MF and umbilical cord blood were immunoselected (CD34 MicroBead Kit, Miltenyi Biotec) according to the manufacturer's instructions. Enrichment for $\mathrm{CD} 4^{+}$cells was ascertained by flow cytometry using an anti-CD34 monoclonal antibody (clone 581, BD Pharmingen). $\mathrm{CD}^{+} 4^{+}$cells were cultured in StemSpan serum-free medium (STEMCELL Technologies) with stem cell factor (SCF) at 20 ng/mL (STEMCELL Technologies).

Primary BM stromal cells. Primary BM stromal cells were obtained from the BM of patients with chronic myelogenous leukemia. Briefly, after immunoselection of the $\mathrm{CD} 34^{+}$population, the $\mathrm{CD} 34^{-}$population was cultured in DMEM/GlutaMAX/10\% FBS without cytokines. Nonadherent cells were removed by successive passages.

Cell lines for proliferation assays. UKE-1 (CVCL_0104) and HEL (CVCL_0001) cells carrying the JAK2 ${ }^{\mathrm{V} 617 \mathrm{~F}}$ mutation were used for proliferation assay and were cultured in RPMI/GlutaMAX/10\% FBS.

$B M$ stromal cell lines. The murine BM stromal cell line MS5 (CVCL_2128) and the human BM stromal cell line HS5 (CVCL_3720) were used to test the antifibrotic effect of PPAR $\gamma$ agonists in vitro and were cultured in DMEM/GlutaMAX/10\% FBS.

\section{Reagents}

For in vitro assays, PPAR $\gamma$ agonists were provided by Cayman Chemical (PPAR $\gamma$ PAK; Bertin-pharma). The specific inhibitor of activin receptor-like kinase (Alk) SB-431542 (4-[4-(1,3-benzodioxol-5-yl)-5(2-pyridinyl)-1H-imidazol-2-yl]-benzamide) was provided by SigmaAldrich and used at a final concentration of $1 \mu \mathrm{M}$. The p300 HAT inhibitor C646 (4-[4-[[5-(4,5-dimethyl-2-nitrophenyl)-2-furanyl] methylene]-4,5-dihydro-3-methyl-5-oxo-1 $\mathrm{H}$-pyrazol-1-yl]benzoic acid) was provided by Sigma-Aldrich and used at a final concentration of $20 \mu \mathrm{M}$. The MEK1/2 inhibitor U0126 [1,4-diamino-2,3-dicyano-1,4-bis-(2-aminophenylthio)-butadiene] was provided by InvivoGen and used at a final concentration of $10 \mu \mathrm{M}$. The JNK inhibitor SP600125 was provided by MedChemExpress (CliniSciences) and used at a final concentration of $20 \mu \mathrm{M}$. Recombinant TGF- $\beta 1$ was provided by R\&D Systems and used at $5 \mathrm{ng} / \mathrm{mL}$.

\section{Proliferation assays}

CD $34{ }^{+}$cells from patients with PV or MF and umbilical cord blood and UKE-1 and HEL cells were cultured with or without pioglitazone (10 or $20 \mu \mathrm{M})$. Cell viability and absolute count were assessed by flow cytometry at days 3,6 , and 10 of culture. 


\section{Induction of TGF- $\beta 1$ target genes in BM stromal cells}

BM stromal cells were stimulated with TGF- $\beta 1(5 \mathrm{ng} / \mathrm{mL})$ with or without pioglitazone (10 or $20 \mu \mathrm{M})$ and with or without chemical inhibitors (SB-431542, C646, SP600125, or U0126). After 5 hours at $37^{\circ} \mathrm{C}$ in $5 \%$ $\mathrm{CO}_{2}$, cells were harvested and suspended in an appropriate lysis buffer for RNA extraction.

\section{Production of the p300 HAT and p300 HAT- lentiviral vectors}

The cDNAs encoding p300 HAT and p300 HAT ${ }^{-}$(mutation: H1415A E1423A Y1424A L1428S Y1430A H1434A) were obtained from Addgene (plasmid 23252 and plasmid 23254, respectively) and subcloned into the pLenti-GIII-CMV-GFP-2A-Puro lentiviral vector provided by Applied Biological Materials Inc. Lentiviral particle production was performed as previously described (21) and the particles concentrated by centrifugation using Vivaspin 20 Ultrafiltration Units (Sartorius).

\section{RNA extraction and quantitative reverse transcription PCR analysis}

RNA was extracted from $2 \times 10^{5}$ cells using RNAqueous-4PCR (Ambion). Reverse transcription was carried out for 1 hour at $42^{\circ} \mathrm{C}$ using the SuperScript VILO cDNA Synthesis kit (Invitrogen Life Technologies) according to the manufacturer's instructions. Real-time PCR was performed in an iCycler thermocycler (CFX, Bio-Rad) using iQ Supermix SYBR GRN (Bio-Rad). The primer pairs targeting the human and murine cDNA are listed in Supplemental Table 3. The comparative CT method $(\triangle \Delta \mathrm{CT})$ was used to compare gene expression levels between the various culture conditions (relative to GAPDH).

\section{Treatment and analysis of mice}

Treatment with PPAR $\gamma$ agonists. Each treatment group comprised 8-10 female mice (C57BL/6J; Charles River Laboratories), regardless of the mouse model. Mice were randomly assigned to the treatment group after transplantation (www.randomization.com). Treatment was given orally in pellets containing pioglitazone (Actos, Takeda) at a dose of $250 \mathrm{mg} / \mathrm{kg}$ of food, pellets containing mesalazine (Sigma-Aldrich) at a dose of $2500 \mathrm{mg} / \mathrm{kg}$ of food, or control pellets (C1000). All pellets came from Genestil. Mice of the $\mathrm{TPO}^{\text {high }}$ model were treated from week 2 until euthanasia at week 15 . MF post-JAK2 ${ }^{\mathrm{V} 617 \mathrm{~F}}$ mice were treated from week 20 until euthanasia at week 37 . JAK2 ${ }^{\mathrm{V} 617 \mathrm{~F}}-\mathrm{GFP}$ mice were treated from week 6 until euthanasia at week 23. CALRdel52 and CALR-WT mice were treated from week 6 until euthanasia at week 20.

Blood samples. Iterative venous blood samples (100 to $200 \mu \mathrm{L})$ were taken from either the retro-orbital sinus or the mandibular vein. All blood counts were performed on a scil Vet abc device. A portion of the cells from the peripheral blood samples were lysed in an appropriate buffer for RNA extraction.

Quantitative determination of TGF- $\beta 1$ concentrations. The quantification of plasma TGF- $\beta 1$ was carried out with the Quantikine ELISA kit (DB100B, R\&D Systems) in accordance with the manufacturer's instructions.

$B M$ samples. The BM cavity of one femur per mouse was rinsed in a constant volume $(100 \mu \mathrm{L})$. Part of the BM cell suspension was labeled with a mixture of monoclonal antibodies and counted by flow cytometry using BD Trucount (BD Biosciences) tubes. Another portion of the BM cells $\left(1 \times 10^{5}\right.$ cells $)$ were suspended in $3 \mathrm{~mL}$ of $\alpha$-MEM-based methylcellulose medium (MethoCult GF M4434, STEMCELL Technologies). Cells were scored and collected after 14 days of incubation at $37^{\circ} \mathrm{C}$ in a $5 \% \mathrm{CO}_{2}$ atmosphere. A portion of the BM cells was depleted of mature cells with a mixture of biotinylated anti-CD5, anti-CD45R (B220), anti-CD11b, anti-Gr1 (Ly-6G/C), anti-CD7-4, and anti-Ter119 antibodies according to the manufacturer's recommendations (Lineage Cell Depletion Kit, mouse; Miltenyi Biotec). The Lin' cells were then labeled with an anti-Sca.1 antibody and an anti-CD117 antibody to identify the so-called LSK fraction. Additional labeling with an anti-CD34 antibody differentiated LSK-CD34+ ${ }^{+}$cells corresponding to short-term hematopoietic stem cells (ST-HSCs) from LSK-CD34- cells corresponding to long-term hematopoietic stem cells (LT-HSCs).

Histopathological analysis of BM and spleen. Femurs and spleens were fixed in formaldehyde, decalcified, and paraffin-embedded. Sections were stained with H\&E, periodic acid-Schiff, and Giemsa for cytology analysis. Reticulin fibers were revealed by silver staining according to Gordon and Sweet's method. Megakaryocytes (MKs) were revealed by histochemistry with a rabbit anti-von Willebrand factor polyclonal antibody (Dako). Images were obtained using a Zeiss Axiophot microscope with a $\times 10$ Zeiss lens, a Zeiss Axiocam MRc camera, and AxioVision release 4.3 acquisition software. MK sizes were calculated using the ImageJ 1.53a image processing program (NIH).

\section{Progenitor cell study}

For human colony-forming cell assays, $1 \times 10^{4} \mathrm{CD} 34^{+}$hematopoietic progenitor cells or 500 cells from the cell lines (UKE-1 or HEL) were suspended in $3 \mathrm{~mL} \alpha$-MEM-based methylcellulose medium (MethoCult GF H4434, STEMCELL Technologies). For murine colony-forming cell assays, $2 \times 10^{5} \mathrm{BM}$ mononuclear cells were suspended in 3 $\mathrm{mL} \alpha$-MEM-based methylcellulose medium (MethoCult GF M4434, STEMCELL Technologies). All colony-forming precursor cells were scored after 14 days of incubation at $37^{\circ} \mathrm{C}$ and $5 \% \mathrm{CO}_{2}$.

\section{Flow cytometry}

Antibodies. Antibodies used for flow cytometry were all purchased from BD Pharmingen and included anti-human CD45 (clone 2D1), CD34 (clone 581), annexin V, and Smad2(pS465/pS467)/Smad3(pS423/ pS425) (clone O72-670, BD Phosflow Smad Monoclonal Antibodies); anti-mouse CD 45 (clone 30-F11), CD45.1 (clone A20), CD45.2 (clone 104), CD34 (clone RAM34), Sca.1 (Ly-6A/E) (clone D7), and CD117 (Kit) (ACK45); and anti-p-STAT5 (pY694). Lineage-negative cell populations from mouse $\mathrm{BM}$ samples $\left(\mathrm{Lin}^{-}\right)$were obtained by depletion of mature hematopoietic cells with the Lineage Cell Depletion Kit (Miltenyi Biotec).

Detection of apoptosis by annexin $V$ staining. Cells $\left(1 \times 10^{5}\right)$ were washed and suspended in binding buffer (0.01 M HEPES/NaOH $[\mathrm{pH}$ 7.4], $0.14 \mathrm{M} \mathrm{NaCl}, 2.5 \mathrm{mM} \mathrm{CaCl}_{2}$ ) before incubation with $5 \mu \mathrm{L}$ antiannexin $\mathrm{V}$-APC for 15 minutes at room temperature $\left(25^{\circ} \mathrm{C}\right)$ in the dark. Then, $400 \mu \mathrm{L}$ binding buffer and $5 \mu \mathrm{L}$ propidium iodide (PI) (component 51-66211E, BD Biosciences) were added 10 minutes before the analysis was performed. The apoptotic population was defined as annexin V-positive and PI-negative cells.

DNA synthesis assay. The cell proliferation rate was measured by incorporation of 5-ethynyl-2'-deoxyuridine (EdU), a thymidine nucleoside analog, into DNA during active DNA synthesis (2 hours). Staining was performed according to the manufacturer's protocol (Click-iT EdU Flow Cytometry Assay Kit, Invitrogen).

Cell cycle analysis. Cells previously stained for the DNA synthesis assay were incubated with Hoechst $33342(10 \mu \mathrm{g} / \mathrm{mL})$ for $15 \mathrm{~min}$ utes at $37^{\circ} \mathrm{C}$ and then centrifuged at $200 \mathrm{~g}$ for 5 minutes and washed 
2 times in PBS. Cells were analyzed by flow cytometry using violet excitation and collecting the emitted Hoechst fluorescence between 390 and $480 \mathrm{~nm}$.

Intracellular Smad2/3 phosphorylation assays. Briefly, MS5 and HS5 cells cultured in DMEM with $10 \%$ FCS were incubated with or without pioglitazone $(10 \mu \mathrm{M})$ and TGF- $\beta 1(5 \mathrm{ng} / \mathrm{mL})$ at $37^{\circ} \mathrm{C}$ in $5 \% \mathrm{CO}_{2}$ for 2 hours. Cells were fixed and permeabilized using Cytofix/Cytoperm 4 (BD Pharmingen) and stained with anti-phospho-Smad2/3 (BD Pharmingen) for 30 minutes in the dark according to the manufacturer's protocol. Analysis was carried out on a minimum of 20,000 events in the viable-cell gate.

For all experiments (except annexin V-based detection of apoptosis and cell cycle analysis), cell viability was assessed using SYTOX Blue dead cell stain (Invitrogen Life Technologies). All analyses were carried out on a BD FACSCanto II flow cytometer.

\section{ChIP assays}

ChIP analysis was performed on BM MS5 cell line after 5 hours of TGF- $\beta 1$ stimulation $(10 \mathrm{ng} / \mathrm{mL})$, with or without pioglitazone (20 $\mathrm{ng} / \mathrm{mL}$ ). Technical details are provided in Supplemental Table 4, and raw data were uploaded to the Gene Expression Omnibus (GEO) site with the accession number GSE141378 (https://www.ncbi.nlm. nih.gov/geo/query/acc.cgi?acc $=$ GSE141378). Nucleotide sequences were mapped using Bowtie (v2.3.4.3) against the mouse genome mm9 [UCSC Genome Browser on Mouse July 2007 (NCBI37/mm9) Assembly] downloaded from the UCSC Genome Browser (http:// genome.ucsc.edu). Then SAM files were converted into BAM format using SAMtools (v1.9). Mapped reads (sequences) were transformed into a genome-wide read density (coverage), in 2 steps: (a) using makeUCSCfile from Homer suite (v4.10, 5-16-2018) with the parameter -norm 20000000 to normalize the number of reads, and (b) using bedGraphToBigWig (v4) from UCSC. Peak calling was performed with Homer (v4.10, 5-16-2018) with default parameters.

\section{Quantification of plasma pioglitazone and mesalazine}

Both compounds were quantified by a method validated by liquid chromatography coupled to tandem mass spectrometry detection (LC-MS/MS). Twenty microliters of plasma was precipitated after addition of internal standard (glyburide-D3 for pioglitazone and metformin-D6 for mesalazine). After centrifugation, $20 \mu \mathrm{L}$ of supernatant was injected into the LC-MS/MS system. Separation was achieved on a Hypersil Gold pentafluorophenyl column $(100 \times 2.1 \mathrm{~mm}$ ID, particle size $1.9 \mu \mathrm{m}$; Thermo Fisher Scientific). The mobile phase was a gradient of acetonitrile (A) and ammonium formate buffer $(2 \mathrm{mmol} / \mathrm{L}$ formate in $0.1 \%$ formic acid) (B) proceeding from $25 \% \mathrm{~A}$ to $90 \% \mathrm{~A}$ in 4.5 minutes and holding for 0.5 minutes, at a flow rate of $400 \mu \mathrm{L} / \mathrm{min}$. The total run time was 5 minutes. Compounds were detected by a TSQ Altis triple-quadrupole mass spectrometer (Thermo Fisher Scientific).
Data were collected in selected reaction monitoring using the transition $m / z 357.2 \rightarrow 119.1$ (quantifying ion [QI]) and 134.2 for pioglitazone and $m / z 152.0 \rightarrow 108.1$ (QI) and 107.1 for mesazaline.

\section{Statistics}

For culture assays and quantitative real-time PCR, values were calculated as the mean \pm SD for at least 3 separate experiments performed in triplicate. Paired and unpaired comparisons were performed using the nonparametric Wilcoxon rank test and Mann-Whitney test, respectively. Levels of significance to correct for multiple comparisons for experiments with more than 2 groups were adjusted using the Holm procedure (80). A $P$ value less than 0.05 was considered significant.

\section{Study approval}

All patient samples were obtained with written informed consent. Animal experiments were approved by the Commissariat à l'Energie Atomique/Direction de la Recherche Fondamentale review board (Fontenay-Aux-Roses, France), protocol 16_032.

\section{Author contributions}

JL, JS, and SP led the project, designed and performed experiments, and analyzed data. WV, IP, JLV, CM, MS, and ES analyzed data. JCA measured plasma concentrations of pioglitazone and mesalazine. MD performed statistical analysis. CC, KSF, and VE contributed experimentally. PR and JJK analyzed data and contributed clinically. JL and SP wrote the manuscript. All authors approved the manuscript.

\section{Acknowledgments}

We thank E. Kouroupi for her clinical contribution. We thank Catherine Lacout (Gustave Roussy) and Milena Kohn for their experimental contributions, Olivia Bawa (Gustave Roussy) for the histology, and Warren Alexander (Walter Elisa Hall Institute, Melbourne, Australia) for the VavCre mice. This work was supported by the Association Laurette Fugain (2017-06), Paris, France (to SP, PR, JJK, and JLV); the Association pour la Recherche sur le Cancer (PJA20161204566), Villejuif, France (to SP); Action Leucémies, Boulogne-Billancourt, France (to JL); the Agence Nationale pour la Recherche (ANR) (HemaPpar) (to SP); and Institut National Du Cancer (INCA) (Plbio 2014 to JLV and JJK, Plbio 2018 to IP and JJK). ES was supported by Fondation pour la Recherche Médicale (Equipe FRM DEQ20180339221), the ATIP-AVENIR program (Plan Cancer), and the Labex EpiGenMed (Investissements d'avenir program, reference ANR-10-LABX-12-01).

Address correspondence to: Stéphane Prost, CEA/DRF/IbFj/IMVAHB, 18 Route du Panorama, 92265 Fontenay-Aux-Roses cedex, France. Phone: 33.1.46.54.94.69; Email: stephane.prost@cea.fr.
1. Tefferi A, Vardiman JW. Classification and diagnosis of myeloproliferative neoplasms: the 2008 World Health Organization criteria and point-of-care diagnostic algorithms. Leukemia. 2008;22(1):14-22.

2. James $\mathrm{C}$, et al. A unique clonal JAK2 mutation leading to constitutive signalling causes polycythaemia vera. Nature. 2005;434(7037):1144-1148.
3. Klampfl T, et al. Somatic mutations of calreticulin in myeloproliferative neoplasms. $N$ Engl JMed. 2013;369(25):2379-2390.

4. Tefferi A. Myelofibrosis with myeloid metaplasia. N Engl JMed. 2000;342(17):1255-1265.

5. Cervantes F, et al. Improving survival trends in primary myelofibrosis: an international study. JClin Oncol. 2012;30(24):2981-2987.
6. Tabarroki A, Tiu RV. Molecular genetics of myelofibrosis and its associated disease phenotypes. Transl Med UniSa. 2014;8:53-64.

7. Vannucchi AM, et al. Philadelphia chromosomenegative chronic myeloproliferative neoplasms: ESMO Clinical Practice Guidelines for diagnosis, treatment and follow-up. Ann Oncol. 2015;26(Suppl 5):v85-v99. 
8. Cervantes F, et al. Alleviating anemia and thrombocytopenia in myelofibrosis patients. Expert Rev Hematol. 2016;9(5):489-496.

9. Harrison C, et al. JAK inhibition with ruxolitinib versus best available therapy for myelofibrosis. N Engl J Med. 2012;366(9):787-798.

10. Passamonti F, Maffioli M. The role of JAK2 inhibitors in MPNs 7 years after approval. Blood. 2018;131(22):2426-2435.

11. Verstovsek S, et al. A double-blind, placebocontrolled trial of ruxolitinib for myelofibrosis. N Engl J Med. 2012;366(9):799-807.

12. Verstovsek $\mathrm{S}$, et al. Long-term survival in patients treated with ruxolitinib for myelofibrosis: COMFORT-I and -II pooled analyses. J Hematol Oncol. 2017;10(1):156.

13. Mead AJ, et al. Response to ruxolitinib in patients with intermediate-1-, intermediate-2-, and highrisk myelofibrosis: results of the UK ROBUST Trial. Br J Haematol. 2015;170(1):29-39.

14. Ianotto JC, et al. Benefits and pitfalls of pegylated interferon- $\alpha 2 \mathrm{a}$ therapy in patients with myeloproliferative neoplasm-associated myelofibrosis: a French Intergroup of Myeloproliferative neoplasms (FIM) study. Haematologica. 2018;103(3):438-446.

15. Hasan S, et al. JAK2V617F expression in mice amplifies early hematopoietic cells and gives them a competitive advantage that is hampered by IFN $\alpha$. Blood. 2013;122(8):1464-1477.

16. Marty C, et al. Calreticulin mutants in mice induce an MPL-dependent thrombocytosis with frequent progression to myelofibrosis. Blood. 2016;127(10):1317-1324.

17. Villeval JL, et al. High thrombopoietin production by hematopoietic cells induces a fatal myeloproliferative syndrome in mice. Blood. 1997;90(11):4369-4383.

18. Dunbar A, et al. Overview of transgenic mouse models of myeloproliferative neoplasms (MPNs). Curr Protoc Pharmacol. 2017;77:14.40.1-14.40.19.

19. Lehrke M, Lazar MA. The many faces of PPARgamma. Cell. 2005;123(6):993-999.

20. Prost $S$, et al. Human and simian immunodeficiency viruses deregulate early hematopoiesis through a Nef/PPARgamma/STAT5 signaling pathway in macaques. J Clin Invest. 2008;118(5):1765-1775.

21. Prost S, et al. Erosion of the chronic myeloid leukaemia stem cell pool by PPAR $\gamma$ agonists. Nature. 2015;525(7569):380-383.

22. Rousselot P, et al. Pioglitazone together with imatinib in chronic myeloid leukemia: a proof of concept study. Cancer. 2017;123(10):1791-1799.

23. Daynes RA, Jones DC. Emerging roles of PPARs in inflammation and immunity. Nat Rev Immunol. 2002;2(10):748-759.

24 . Ricote $\mathrm{M}$, et al. The peroxisome proliferator-activated receptor-gamma is a negative regulator of macrophage activation. Nature. 1998;391(6662):79-82.

25. Jiang $C$, et al. PPAR-gamma agonists inhibit production of monocyte inflammatory cytokines. Nature. 1998;391(6662):82-86.

26. Akbiyik F, et al. Human bone marrow megakaryocytes and platelets express PPARgamma, and PPARgamma agonists blunt platelet release of CD40 ligand and thromboxanes. Blood.
2004;104(5):1361-1368.

27. Dantas AT, et al. The role of PPAR gamma in systemic sclerosis. PPAR Res. 2015;2015:124624.

28. Agarwal A, et al. Bone marrow fibrosis in primary myelofibrosis: pathogenic mechanisms and the role of TGF- $\beta$. Stem Cell Investig. 2016;3:5.

29. Biernacka $A$, et al. TGF- $\beta$ signaling in fibrosis. Growth Factors. 2011;29(5):196-202.

30. Pardali E, et al. TGF- $\beta$-induced endothelial-mesenchymal transition in fibrotic diseases. Int J Mol Sci. 2017;18(10):E2157.

31. Yue L, et al. Efficacy of ALK5 inhibition in myelofibrosis. JCI Insight. 2017;2(7):e90932.

32. Dong M, Blobe GC. Role of transforming growth factor-beta in hematologic malignancies. Blood. 2006;107(12):4589-4596

33. Terui $\mathrm{T}$, et al. The production of transforming growth factor-beta in acute megakaryoblastic leukemia and its possible implications in myelofibrosis. Blood. 1990;75(7):1540-1548.

34. Martyre MC, et al. Increased intraplatelet levels of platelet-derived growth factor and transforming growth factor-beta in patients with myelofibrosis with myeloid metaplasia. Br J Haematol. 1991;77(1):80-86.

35. Chagraoui $\mathrm{H}$, et al. Prominent role of TGF-beta 1 in thrombopoietin-induced myelofibrosis in mice. Blood. 2002;100(10):3495-3503.

36. Benlabiod C, et al. Calreticulin del52 and ins5 knock-in mice recapitulate different myeloproliferative phenotypes observed in patients with MPN. Nat Commun. 2020;11(1):4886.

37. Berria R, et al. Reduction in hematocrit and hemoglobin following pioglitazone treatment is not hemodilutional in Type II diabetes mellitus. Clin Pharmacol Ther. 2007;82(3):275-281.

38. Yan D, et al. Critical requirement for Stat 5 in a mouse model of polycythemia vera. Blood. 2012;119(15):3539-3549.

39. Mondet J, et al. Circulating cytokine levels as markers of inflammation in Philadelphia negative myeloproliferative neoplasms: diagnostic and prognostic interest. Mediators Inflamm. 2015;2015:670580.

40. Dalen KT, et al. Adipose tissue expression of the lipid droplet-associating proteins S3-12 and perilipin is controlled by peroxisome proliferator-activated receptor-gamma. Diabetes. 2004;53(5):1243-1252.

41. Perrier S, et al. IL-1 receptor antagonist in metabolic diseases: Dr Jekyll or Mr Hyde? FEBS Lett. 2006;580(27):6289-6294.

42. Duncan MR, et al. Connective tissue growth factor mediates transforming growth factor betainduced collagen synthesis: down-regulation by cAMP. FASEB J. 1999;13(13):1774-1786.

43. Mori T, et al. Role and interaction of connective tissue growth factor with transforming growth factor-beta in persistent fibrosis: a mouse fibrosis model. J Cell Physiol. 1999;181(1):153-159.

44. Ghosh AK, et al. Peroxisome proliferatoractivated receptor-gamma abrogates Smaddependent collagen stimulation by targeting the p300 transcriptional coactivator. FASEB J. 2009;23(9):2968-2977.

45. Zhu M, et al. Anti-inflammatory effects of thiazolidinediones in human airway smooth muscle cells. Am J Respir Cell Mol Biol. 2011;45(1):111-119.
46. Hussein K, et al. Cytokine expression pattern in bone marrow microenvironment after allogeneic stem cell transplantation in primary myelofibrosis. Biol Blood Marrow Transplant. 2016;22(4):644-650.

47. Kroger N, et al. Impact of allogeneic stem cell transplantation on survival of patients less than 65 years of age with primary myelofibrosis. Blood. 2015;125(21):3347-3350.

48. Guo B, et al. Antagonism of PPAR- $\gamma$ signaling expands human hematopoietic stem and progenitor cells by enhancing glycolysis. Nat Med. 2018;24(3):360-367.

49. Sertorio M, et al. In vivo RNAi screen unveils PPAR $\gamma$ as a regulator of hematopoietic stem cell homeostasis. Stem Cell Reports. 2017;8(5):1242-1255.

50. Garbati MR, et al. Mutant calreticulin-expressing cells induce monocyte hyperreactivity through a paracrine mechanism. Am J Hematol. 2016;91(2):211-219.

51. Rampal R, et al. Integrated genomic analysis illustrates the central role of JAK-STAT pathway activation in myeloproliferative neoplasm pathogenesis. Blood. 2014;123(22):e123-e133.

52. Geduk A, et al. The role of $\beta$-catenin in $\mathrm{Bcr} /$ Abl negative myeloproliferative neoplasms: an immunohistochemical study. Clin Lymphoma Myeloma Leuk. 2015;15(12):785-789.

53. Suboticki T, et al. $\beta$-Catenin and PPAR- $\gamma$ levels in bone marrow of myeloproliferative neoplasm: an immunohistochemical and ultrastructural study. Ultrastruct Pathol. 2018;42(6):498-507.

54. Heaton WL, et al. Autocrine Tnf signaling favors malignant cells in myelofibrosis in a Tnfr2-dependent fashion. Leukemia. 2018;32(11):2399-2411.

55. Carobbio A, et al. Leukocytosis and thrombosis in essential thrombocythemia and polycythemia vera: a systematic review and meta-analysis. Blood Adv. 2019;3(11):1729-1737.

56. Rumi E, Cazzola M. Diagnosis, risk stratification, and response evaluation in classical myeloproliferative neoplasms. Blood. 2017;129(6):680-692.

57. Falanga A, et al. V617F JAK-2 mutation in patients with essential thrombocythemia: relation to platelet, granulocyte, and plasma hemostatic and inflammatory molecules. Exp Hematol. 2007;35(5):702-711.

58. Fleischman AG. Inflammation as a driver of clonal evolution in myeloproliferative neoplasm. Mediators Inflamm. 2015;2015:606819.

59. Schepers K, et al. Myeloproliferative neoplasia remodels the endosteal bone marrow niche into a self-reinforcing leukemic niche. Cell Stem Cell. 2013;13(3):285-299.

60. Williams C, et al. Optimizing clinical use of mesalazine (5-aminosalicylic acid) in inflammatory bowel disease. Therap Adv Gastroenterol. 2011;4(4):237-248.

61. Palma A, et al. Peroxisome proliferator-activated receptor-gamma expression in monocytes/ macrophages from rheumatoid arthritis patients: relation to disease activity and therapy efficacy-a pilot study. Rheumatology (Oxford). 2012;51(11):1942-1952.

62. Croasdell A, et al. PPAR $\gamma$ and the innate immune system mediate the resolution of inflammation. 
PPAR Res. 2015;2015:549691.

63. Celinski K, et al. Effects of peroxisome proliferator-activated receptors-gamma ligands on dextran sodium sulphate-induced colitis in rats. JPhysiol Pharmacol. 2011;62(3):347-356.

64. Cuzzocrea S, et al. Rosiglitazone and 15-deoxy- $\Delta^{12,14}$-prostaglandin $\mathrm{J}_{2}$, ligands of the peroxisome proliferator-activated receptor- $\gamma$ (PPAR- $\gamma$ ), reduce ischaemia/reperfusion injury of the gut. Br J Pharmacol. 2003;140(2):366-376.

65. Zhang $\mathrm{Q}$, et al. PPAR $\gamma$ agonist rosiglitazone is neuroprotective after traumatic spinal cord injury via anti-inflammatory in adult rats. Neurol Res. 2010;32(8):852-859.

66. Lakshmi SP, et al. Transforming growth factor $\beta$ suppresses peroxisome proliferator-activated receptor $\gamma$ expression via both SMAD binding and novel TGF- $\beta$ inhibitory elements. Biochem J. 2017;474(9):1531-1546.

67. Lee SJ, et al. Peroxisome proliferator-activated receptor-gamma and retinoic acid $\mathrm{X}$ receptor alpha represses the TGFbeta1 gene via PTEN-mediated p70 ribosomal S6 kinase-1 inhibition: role for Zf9 dephosphorylation. Mol Pharmacol. 2006;70(1):415-425.

68. Teixeira AF, et al. On-target anti-TGF- $\beta$ therapies are not succeeding in clinical cancer treatments: what are remaining challenges? Front Cell Dev Biol. 2020;8:605.

69. Hou Y, et al. PPAR $\gamma$ is an E3 ligase that induces the degradation of NFkB/p65. Nat Commun. 2012;3:1300.

70. Scirpo R, et al. Stimulation of nuclear receptor peroxisome proliferator-activated receptor- $\gamma$ limits NF-кB-dependent inflammation in mouse cystic fibrosis biliary epithelium. Hepatology. 2015;62(5):1551-1562.

71. Jacobs MD, Harrison SC. Structure of an IkappaBalpha/NF-kappaB complex. Cell. 1998;95(6):749-758.

72. Glatz T, et al. Peroxisome-proliferator-activated receptors gamma and peroxisome-proliferatoractivated receptors beta/delta and the regulation of interleukin 1 receptor antagonist expression by pioglitazone in ischaemic brain.

JHypertens. 2010;28(7):1488-1497.

73. Vetuschi A, et al. PPAR- $\gamma$ with its anti-inflammatory and anti-fibrotic action could be an effective therapeutic target in IBD. Eur Rev Med Pharmacol Sci. 2018;22(24):8839-8848.

74. Wei J, et al. Regulation of matrix remodeling by peroxisome proliferator-activated receptor- $\gamma$ : a novel link between metabolism and fibrogenesis. Open Rheumatol J. 2012;6:103-115.

75. Wei J, et al. Peroxisome proliferator-activated receptor $\gamma$ : innate protection from excessive fibrogenesis and potential therapeutic target in systemic sclerosis. Curr Opin Rheumatol. 2010;22(6):671-676.

76. Yang L, et al. Regulation of peroxisome proliferator-activated receptor-gamma in liver fibrosis. Am J Physiol Gastrointest Liver Physiol. 2006;291(5):G902-G911.

77. Ghosh AK, et al. Constitutive Smad signaling and Smad-dependent collagen gene expression in mouse embryonic fibroblasts lacking peroxisome proliferator-activated receptor-gamma. Biochem Biophys Res Commun. 2008;374(2):231-236.

78. Zhao C, et al. PPARgamma agonists prevent TGFbeta1/Smad3-signaling in human hepatic stellate cells. Biochem Biophys Res Commun. 2006;350(2):385-391.

79. Ge C, et al. Reciprocal control of osteogenic and adipogenic differentiation by ERK/MAP kinase phosphorylation of Runx2 and PPAR $\gamma$ transcription factors. JCell Physiol. 2016;231(3):587-596.

80. Holm S. A simple sequentially rejective multiple test procedure. Scand J Stat. 1979;6:65-70. 